\title{
Towards Exact Quantum Loop Results in the Theory of General Relativity
}

\author{
B.F.L. Ward*
}

Department of Physics, Baylor University, Waco, Texas, 76798-7316, USA

\begin{abstract}
We show that, by using resummation techniques based on the extension of the methods of Yennie, Frautschi and Suura to Feynman's formulation of Einstein's theory, we get calculable loop corrections that are even (superficially) free of UV divergences. The UV finiteness of the loops results from resumming large IR terms $\mathcal{O}\left(G_{N}\left|k^{2}\right| \ln \left|k^{2}\right|\right)^{n}, n=1, \ldots$, $\infty$ for $\left|\mathrm{k}^{2}\right| \rightarrow \infty$, where $G_{N}$ is Newton's constant. We illustrate our results with applications of some phenomenological interest.
\end{abstract}

\section{INTRODUCTION}

The many successes of Einstein's classical theory of general relativity are well-known $[1,2]$. Given the outstanding success of the Standard Model (SM) [3-12] point particle quantum field theory for the other three known forces, the electromagnetic, weak and strong interactions, where the non-Abelian loop corrections predicted by the 't HofftVeltman [6-9] renormalization theory for Yang-Mills fields [13] have recently been corroborated by the precision SM tests [14-16] at the CERN LEP Collider, we have to agree that the union of quantum mechanics and the classical theory of general relativity is one key piece of unfinished business left-over for the $21 \mathrm{st}$ century. At this writing, the only accepted complete treatment of quantum general relativity, superstring theory $[17,18]$, involves $[19-21]^{1}$ many hitherto unseen degrees of freedom, some at masses well-beyond the Planck mass, and this latter property is understandably a bit unsettling to some. Is it possible that such degrees of freedom are anything more than a mathematical artifact?

Why can we not apply the 't Hofft-Veltman calculus for non-Abelian loop corrections to quantum general relativity (QGR)? After all, the Feynman-Faddeev-Popov ghost field technique, so crucial to the 't Hooft-Veltman renormalization program, was invented by Feynman $[22,23]$ in his pioneering work on Einstein's theory. Is it really true, as Einstein suggested, that Bohr's quantum mechanics is just too incomplete to include general relativity in its domain of applicability? The superstring theory $[17,18]$ candidate approach to quantum general relativity would suggest this as well, as one of its predictions is that one of the basic results in quantum mechanics, the Heisenberg uncertainty principle, is in fact modified [24]. Here, we take a different view which

*Address correspondence to this author at the Department of Physics, Baylor University, Waco, Texas, 76798-7316, USA; E-mail: BFL_Ward@baylor.edu

\footnotetext{
${ }^{1}$ Recently, the loop quantum gravity approach [19-21] has been advocated by several authors, but it has still unresolved theoretical issues of principle, unlike the superstring theory. Like the superstring theory, loop quantum gravity introduces a fundamental length, the Planck length, as the smallest distance in the theory. This is a basic modification of Einstein's theory.
}

we base on the original work of Feynman [22, 23]. The idea is that the graviton field should be treated as any other point particle field in the successful SM theory. Just like the famous Higgs field, which has a non-zero vacuum expectation value about which the physical Higgs field executes quantum fluctuations, so too the graviton field, the metric tensor $g_{\mu v}$, has a vacuum expectation value, which we will take following Feynman to be the Minkowski value $\eta_{\mu v}$, about which the physical graviton particle executes quantum fluctuations. When these fluctuations are large, the quantum fluctuations dominate the metric of space-time and give rise to a regime that has been called a space-time foam [25]. We do not discuss this regime in what follows. When the graviton field fluctuations are small, we expect to be able to calculate perturbatively in them using the standard FeynmanSchwinger-Tomonaga methods if we can find the appropriate representation of the corresponding Feynman series. It is in finding the latter representation that we extend the pioneering ideas of Feynman in our approach.

Our basic objective is to use resummation of large higher order effects to cure the bad UV behavior of Einstein's theory as formulated by Feynman. There are essentially two kinds of resummation algebras that have had some significant amount of success in the precision theoretical work used in comparing the SM predictions with the precision LEP data. In the first kind, at each order in the perturbative expansion, only the terms which are being resummed are retained, so that what one gets is the exact lowest order term and the resummation of the large terms from each order of the loop expansion. While the result is an improvement over the lowest order term, it is in trinsically an approximate expression. We call such a resummation an "approximate resummation". Examples are the results in Refs. [26-32]. The second type of resummation that has proved useful in precision SM physics has the property that, while one isolates the terms to be resummed order by order in perturbation theory, one does not drop the residual terms in those orders so that one ends up with an exact expression in which some or all large terms from each order of perturbation theory are resummed. We call this an "exact resummation". It is an exact re-arrangement of the original Feynman series. Examples are the theory of Yennie, Frautschi and Suura for QED in Ref. [33, 34], its extension 
to Monte Carlo event generators in Refs. [35-54], and the $\mathrm{QCD}$ and $\mathrm{QED} \otimes \mathrm{QCD}$ exponentiation in Refs. [55-65], which are extensions of the YFS theory to non-Abelian gauge theories. It is this latter type of resummation which we employ for QGR here; for, we do not wish to drop any of the effects in theory. For the record, the results in Refs. [26-32, 35-54] have played significant roles in precision tests of SM physics.

There are good physical reasons why resummation of the YFS type properly extended to quantum general relativity may help to tame the bad UV behavior of the latter theory. Indeed, this at first sight might even seem counter-intuitive, as the YFS type of resummation resums large infrared (IR), large distance, effects and the bad UV behavior of quantum general relativity is characteristic of the short distance behavior of the theory. We make two observations here. First, in the propagation of a particle between a point $\mathrm{x}$ and another point $x^{\prime}$ in the deep Euclidean regime, the effective mass squared involved in that propagation is large and negative, turning the normally attractive Newtonian force for large positive masses into a large repulsion - we expect such repulsion to cause the attendant propagation to be damped severely in the exact solutions of the theory. While we can not solve the theory exactly, if we can re-arrange the Feynman series by resumming a dominant part of the large repulsion we can hope to improve greatly the convergence properties of the Feynman series. Second, in the Feynman loop integration in 4-momentum space, there are three regimes in which we may obtain the big logs that represent dominant behavior: the collinear (CL), infrared (IR) and ultra-violet (UV) regimes. The CL regime is definitely important but even in Abelian gauge theory we know that it is difficult to resum into a simple closed form result with exact residuals. The UV regime carries the renormalization algebra for the theory and will, after being tamed, provide us with the relationship between the bare and physical parameters of the theory. Thus, we do not wish to resum the UV regime. This leaves us the IR regime, for which we do have a representation, that of the YFS-type, which is an exact re-arrangement with closed-form results. We can hope that these resummed 1PI vertices will result in an improved convergence of the theory. Indeed, in Ref. [33, 34], it has already been pointed-out that YFS resummation in QED leads to improved UV behavior for the fermion two-point Green function. Here, we exploit this phenomenon applied to quantum general relativity; for, as gravity couples in the IR regime the same way to all particles, we can hope that the improvement we find will apply to all particles' two-point functions. It will be seen that our improvement in the UV results from resumming large IR terms $\mathcal{O}\left(G_{N}\left|k^{2}\right| \ln \left|k^{2}\right|\right)^{n} n \geq$ 0 for $\left|k^{2}\right| \rightarrow \infty$.

We need to add a word of caution to the reader before that reader proceeds. Just as what happens in the famous case of asymptotic freedom [10-12], when one resums the large terms, such as those we have just noted, in a perturbation series to obtain a more convergent series in terms of the attendant resummed expansion units, the effective coupling in QCD and what will be our resumed propagators here, one has no guarantee that the new series, though it is superficially more convergent, is in fact any better behaved when one sums the entire series - both the unresummed series and the resummed series may be asymptotic, so that the true behavior of the functional corresponding to them may be yet different than either. In the QCD case, experiment [66,67] has verified that the series with the vanishing running coupling is seen in Nature. In QGR, as yet we have no such analogous data to support our resummed series. Since we do not have a constructive realization of the quantum field theory that we study, we can not prove that having order-by-order UV finiteness means that the theory is actually UV finite when summed to all orders, as the sum may still diverge. It is for this reason that we say we have discovered a supperficially UV finite approach to QGR.

We recall for reference that, as pointed-out in Ref. [68], there are four basic approaches to the bad UV behavior of QGR:

- extended theories of gravitation such as super symmetric theories (superstrings and supergravity [69]) and loop quantum gravity;

- resummation, a new version of which we discuss presently;

- composite gravitons; and,

- asymptotic safety - fixed point theory, recently pursued with success in Refs. [70-74].

Our approach will allow us to make contact both with the extended theories and with the phenomenological asymptotic safety approach results in Refs. [70-74]. Moreover, we note that the recent results in Refs. [75-85] on the large distance behavior of QGR are not inconsistent with our approach just as chiral perturbation theory in QCD is not inconsistent with the application of perturbative QCD to short distance QCD effects.

Ultimately, any approach to QGR has to confront experimental tests for confirmation. In this paper, we will start this process by addressing some issues in black hole physics, culminating with an answer to the fate of the final state of Hawking [86-88] radiation by an originally massive black hole. These 'tests' give us some confidence that our methods may indeed represent a pure union ${ }^{2}$ of the ideas of Bohr and Einstein, a union which is not in any contradiction with any well-established experimental or theoretical result.

We shall use resummation based on the extension to quantum general relativity of the theory developed by Yennie, Frautschi and Suura (YFS) [33, 34] originally for QED. In Refs. [35-54], we have extended the YFS methods to the SM electroweak theory and used these extended methods to achieve high precision predictions for SM processes at LEP1 and LEP2, which have played important roles in the precision SM tests of the electroweak theory [1416]. Recently [55-65], we have made a preliminary extension of the YFS methods to soft gluon effects in the QCD sector of the SM, with an eye toward the high energy processes at the LHC. In this paper, we extend the YFS methods to treat the bad UV behavior of quantum general relativity.

\footnotetext{
${ }^{2}$ We do not modify Einstein's theory at all. In this way, we differ from the currently practiced superstring theory $[17,18]$ and loop quantum gravity [19-21] approaches to the bad ultra-violet behavior of quantum general relativity. If we are successful, it would be a true union of the original ideas of Bohr and Einstein. We believe this warrants the further study of our approach in its own right.
} 
More specifically, in Refs. [89-97], we have presented initial discussions of our new approach. Here we present the detailed extensions and the complete derivations as needed of the results in Refs. [89-97], as well as several new results. To make this paper self-contained, we start with the defining Einstein Lagrangian as formulated by Feynman in Refs [22, 23] in the context of the Standard Model. This we do in the next section. In Section 3, we develop and explain the extension of the resummation theory of Yennie, Frautschi and Suura to quantum general relativity. In Section 4, we work out some of the implications of our new approach to quantum general relativity and make contact with related work in the literature. Section 5 contains our summary remarks and our outlook. Technical details are relegated to the Appendices.

\section{EINSTEIN'S THEORY AS FORMULATED BY FEYNMAN}

In this section, we formulate Einstein's theory following the approach of Feynman. This will allow us to set our notation and conventions and to reveal the true issues one confronts in quantizing the general theory of relativity.

More precisely, if we denote by $L_{S M}^{\mathcal{G}}(x)$ the generally covariant Standard Model Lagrangian of the electroweak and strong interactions, then the theory of the currently known elementary particle interactions has the point particle field theory Lagrangian

$$
\mathcal{L}(x)=-\frac{1}{2 \kappa^{2}} \sqrt{-g} R+\sqrt{-g} L_{S M}^{\mathcal{G}}(x)
$$

where $R$ is the curvature scalar, $-g$ is the negative of the determinant of the metric of space-time $g_{\mu v}, \kappa=\sqrt{8 \pi G_{N}}$ $\equiv \sqrt{8 \pi / M_{P l}^{2}}$, where $G_{N}$ is Newton's constant, and the SM Lagrangian density, $L_{S M}^{\mathcal{G}}(x)$, which is well-known (see for example, Ref. [3-12, 98]) when invariance under local Poincare symmetry is not required, is readily obtained from the familiar SM Lagrangian density as follows: since $\partial_{\mu} \phi(x)$ is already generally covariant for any scalar field $\phi$ and since the only derivatives of the vector fields in the SM Lagrangian density occur in their curls, $\partial_{\mu} A_{v}^{J}(x)-\partial_{v} A_{\mu}^{J}(x)$, which are also already generally covariant, we only need to give a rule for making the fermionic terms in usual SM Lagrangian density generally covariant. For this, we introduce a differentiable structure with $\left\{\xi^{a}(x)\right\}$ as locally inertial coordinates and an attendant vierbein field $e_{\mu}^{a} \equiv$ $\partial \xi^{a} / \partial x^{\mu}$ with indices that carry the vector representation for the flat locally inertial space, a, and for the manifold of space-time, $\mu$, with the identification of the space-time base manifold metric as $g_{\mu \nu}=e_{\mu}^{a} e_{a v}$ where the flat locally inertial space indices are to be raised and lowered with Minkowski's metric $\eta_{a b}$ as usual. Associating the usual Dirac gamma matrices $\left\{\gamma_{a}\right\}$ with the flat locally inertial space at $\mathrm{x}$, we define base manifold Dirac gamma matrices by $\Gamma_{\mu}(x)=$ $e_{\mu}^{a}(x) \gamma_{a}$. Then the spin connection, $\omega_{\mu^{b}}^{a}=\frac{1}{2} e^{a v}\left(\partial_{\mu} e_{v}^{b}-\partial_{\nu} e_{\mu}^{b}\right)+$ $\frac{1}{2} e^{b_{v}}\left(\partial_{\mu} e_{v}^{a}-\partial_{\nu} e_{\mu}^{a}\right)+\frac{1}{2} e^{a \rho} e^{b \sigma}\left(\partial_{\rho} e_{c_{\sigma}}-\partial_{\sigma} e_{c_{\rho}}\right) e_{\mu}^{c}$ when there is no torsion, allows us to identify the generally covariant Dirac operator for the SM fields by the substitution $i \not \supset \rightarrow$ $i \Gamma(x)^{\mu}\left(\partial_{\mu}+\frac{1}{2} \omega_{\mu b}^{a} \sum_{a}^{b}\right)$, where we have $\sum_{a}^{b}=\frac{1}{4}\left[\begin{array}{ll}\gamma^{b} & \gamma_{a}\end{array}\right]$ everywhere in the SM Lagrangian density. This will generate $L_{S M}^{\mathcal{G}}(x)$ from the usual SM Lagrangian density $L_{S M}(x)$ as it is given in Refs. [3-12, 98], for example. The Lagrangianin (1) will now be treated following the pioneering work of Feynman $[22,23]$.

First, we note that, although the SM Lagrangian is known to contain many point particle fields, as we are studying the basic interplay between quantum mechanics and general relativity, for pedagogical reasons, we focus the the simplest aspect/ of $L_{S M}^{\mathcal{G}}(x)$, namely that part which involves the massive spinless physical Higgs particle with only its gravitational interactions -it will presumably be observed directly at the LHC in the near future $[99,100]$. The major difficulties in developing a consistent quantum theory of general relativity are all present in this simplification of (1), as has been emphasized by Feynman [22, 23]. We can return to the treatment of the rest of (1) elsewhere.

In this way we are led to consider here the same theory studied by Feynman in Refs. [22, 23],

$$
\begin{aligned}
\mathcal{L}(x)= & -\frac{1}{2 \kappa^{2}} R \sqrt{-g}+\frac{1}{2}\left(g^{\mu \nu} \partial_{\mu} \varphi \partial_{\nu} \varphi-m_{o}^{2} \varphi^{2}\right) \sqrt{-g} \\
= & \frac{1}{2}\left\{h^{\mu \nu, \lambda} \bar{h}_{\mu \nu, \lambda}-2 \eta^{\mu \mu^{\prime}} \eta^{\lambda \lambda^{\prime}} \bar{h}_{\left.\mu_{\lambda, \lambda}, \eta^{\sigma \sigma^{\prime}} \bar{h}_{\mu^{\prime} \sigma, \sigma^{\prime}}\right\}}\right. \\
& +\frac{1}{2}\left\{\varphi_{, \mu} \varphi^{, \mu}-m_{o}^{2} \varphi^{2}\right\}-\kappa h^{\mu \nu}\left[\overline{\varphi_{, \mu} \varphi, \nu}+\frac{1}{2} m_{o}^{2} \varphi^{2} \eta_{\mu \nu}\right] \\
- & \kappa^{2}\left[\frac{1}{2} h_{\lambda \rho} \bar{h}^{\rho \lambda}\left(\varphi_{, \mu} \varphi^{, \mu}-m_{o}^{2} \varphi^{2}\right)-2 \eta_{\rho \rho^{\prime}} h^{\mu \rho} \bar{h}^{\rho^{\prime} \nu} \varphi_{, \mu} \varphi_{, \nu}\right]+\cdots
\end{aligned}
$$

Here, $\varphi(x)$ is the physical Higgs field as our representative scalar field for matter, $\varphi(x)_{, \mu} \equiv \partial_{\mu} \varphi(x)$, and $g_{\mu v}(x)=\eta_{\mu v}+$ $2 \kappa h_{\mu v}(x)$ where we follow Feynman and expand about Minkowski space so that $\eta_{\mu v}=\operatorname{diag}\{1,-1,-1,-1\}$. Following Feynman, we have introduced the notation $\bar{y}_{\mu \nu} \equiv$ $\frac{1}{2}\left(y_{\mu v}+y_{v \mu}-\eta_{\mu v} y_{\rho}{ }^{\rho}\right)$ for any tensor $y_{\mu v}{ }^{4 .}$ Thus, $m_{o}$ is the bare mass of our free Higgs field and we set the small tentatively observed [101] value of the cosmological constant to zero so that our quantum graviton has zero rest mass. We return to this point, however, when we discuss phenomenology. The Feynman rules for (2) have been essentially worked out by Feynman [22, 23], including the rule for the famous Feynman-Faddeev-Popov [22, 102, 103] ghost contribution that must be added to it to achieve a unitary theory with the fixing of the gauge (we use the gauge of Feynman in Ref. [22], $\partial^{\mu} \bar{h}_{v \mu}=0$ ), so we do not repeat this material here. We turn instead directly to the issue of the effect of quantum loop corrections in the theory in (2).

\footnotetext{
${ }^{3}$ This is in preparation by the author.

${ }^{4}$ Our conventions for raising and lowering in dices in the second line of (2) are the same as those in Ref. [23].
} 


\section{RESUMMATION THEORY FOR QUANTUM GENERAL RELATIVITY}

In this section, we develop the resummation theory which we wish to employ in the context of quantum general relativity. We will follow the approach of Yennie, Frautschi and Suura in Ref. [35-54]. This choice is made possible by the formulation of Feynman for Einstein's theory, as the entire theory is a local, point particle field theory, albeit with an infinite number of interaction vertices. Perturbation theory methods can be relevant because, to any finite order in the respective Feynman series, only a finite number of these interaction terms can contribute.

For the scalar field in (2), consider the contributions to the 1PI 2-point function illustrated in Fig. (1). We would like to take advantage of the following physical effect that is intrinsic in Einstein's formulation of Newton's law: for large Euclidean momenta, where the squared momentum transfer in Fig. (1) has a large negative value, the gravitational self energy from Newton's law is strongly repulsive, so that propagation of the particle in this regime should be severely damped in the exact solutions of the theory in (2). This is an intuitive explanation for the success of Weinberg's asymptotic safety approach as recently realized phenomenologically in Refs. [70-74] and leads us to try to resum the large parts of the quantum gravitational loop corrections in order to improve the convergence of the respective Feynman series.

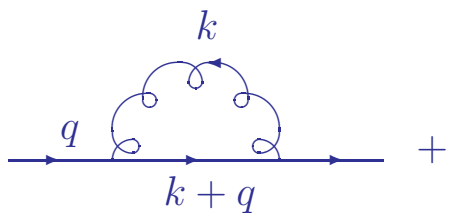

(a)

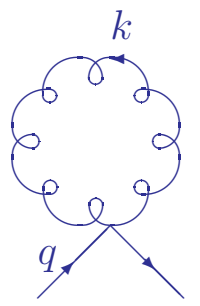

(b)
Fig. (1). Graviton loop contributions to the scalar propagator. $q$ is the 4-momentum of the scalar.

We, however, do not wish to drop-out pieces of this Feynman series. We wish to make an exact re-arrangement of the series in which some of the large gravitational quantum loop effects are resummed to all orders in the loop expansion. Which large gravitational effects shall weresum? In the general Feynman one-loop integral, enhanced contributions arise from three regimes:

- the ultra-violet regime

- the collinear regime

- the infrared regime

The ultra-violet regime will be treated by the renormalization program which we seek to establish here. The collinear regime has been addressed in non-Abelian gauge theories by many authors [104] and we would expect to be able to apply the respective methods to the improved loop expansion that we seek to establish here as well. These methods are as yet generally approximate in the sense of our discussion, they are generically not exact re-arrangements of the Feynman series. We thus look to the infrared regime, for which exact re-arrangement of the loop expansion has been achieved by Yennie, Frautschi and Suura (YFS) in Ref. [33, 34] for Abelian mass less gauge theories. In Refs. [55-61], we have shown that the YFS methods can be extended to non-Abelian gauge theories with the understanding that only the leading IR singular terms actually exponentiate in the YFS sense and that the remaining non-leading and genuinely non-Abelian IR singular terms are treated order by order in the loop expansion. Physically, resummimg this leading IR singular part of the loop expansion in quantum general relativity offers the possibility of improving the convergence of the resummed loop expansion and curing the long standing problem of the non-renormalizability of Einstein's theory. That is what we will argue actually happens in the following. Here, we remind the reader of our cautionary paragraph in the Introduction.

We note here that, already in Ref. [33, 34], it has been pointed-out that YFS resummation of the IR effects in QED improves the UV convergence for the Feynman series for QED. This occurs for the electron propagator but not for the photon propagator and, as the coupling parameter in the soft regime is just $\alpha_{e m} \cong \frac{1}{137}$, the improvement in the convergence via the electron propagator is very marginal, for it is the asymptotic behavior $\frac{1}{p^{1+\mathcal{O}\left(\alpha_{e m}\right)}}$ vs $\frac{1}{p}$ in the deep Euclidean regime. For quantum general general relativity, we will see below that all particles' propagators are improved and that the improvement becomes pronounced in the deep Euclidean regime and causes all propagators to fall faster than any power of the respective momentum transfer $p$.

Returning to Fig. (1), we write the respective contributions to the 1PI proper 2-point vertex function, $-i \Sigma(p)$, the proper self-energy contribution to inverse propagator here, as

$-i\left(\Delta_{F}(p)^{-1}-\Sigma(p)\right)=i \sum_{n=o}^{\infty} \Sigma_{n}(p)$

where $-i \Sigma_{n}(p)$ is the respective n-loop contribution with the agreement that for $n=0$ we have $-\Sigma_{0}(p)=\Delta_{F}(p)^{-1}=p^{2}-m^{2}+$ $i \varepsilon$. For the latter n-loop contribution, we first represent it as follows:

$i \Sigma_{n}(p)=\frac{1}{n !} \int \cdots \int \prod_{i=1}^{n} \frac{d k_{i}}{k_{i}^{2}-\lambda^{2}+i \epsilon} \rho_{n}\left(k_{1}, \cdots, k_{n}\right)$

where the function $\rho_{n}$ is symmetric under the interchange of any two of the $n$ virtual graviton 4-momenta that are exchanged in (4), by the Bose symmetry obeyed by the spin 2 gravitons and the symmetry of the respective multiple integration volume. Here is the point in the discussion where the power of exact rearrangement techniques such as those in Ref. [33, 34] enters. For the case $n=1$, let $s_{g}^{\prime \prime}(k) \rho_{0}$ represent the leading contribution in the limit $k \rightarrow 0$ to $\rho_{1}$. We have

$\rho_{1}(k)=S_{g}^{\prime \prime}(k) \rho_{0}+\beta_{1}(k)$

where this equation is exact and serves to define $\beta_{1}$ if we specify $S_{g}^{\prime \prime}(k)$, the soft graviton emission factor, and recall that 


$$
\rho_{0}=i \Sigma_{0}(p)=-i \Delta_{F}(p)^{-1} .
$$

This can be determined from the Feynman rules for (2) or one can also use the off-shell extension of the formulas in Ref. $[105,106]$. We get [89]

$$
\begin{aligned}
S_{g}^{\prime \prime}(p, p, k) & =\left.\frac{1}{(2 \pi)^{4}} \frac{i \frac{1}{2}\left(\eta^{\mu \nu} \eta^{\bar{\mu} \bar{\nu}}+\eta^{\mu \bar{\nu}} \eta^{\bar{\mu} \nu}-\eta^{\mu \bar{\mu}} \eta^{\nu \bar{\nu}}\right)\left(-i \kappa p_{\bar{\mu}}\right)\left(2 i p_{\mu}\right)\left(-i \kappa p_{\bar{\nu}}^{\prime}\right)\left(2 i p_{\nu}^{\prime}\right)}{\left(k^{2}-2 k p+\Delta+i \epsilon\right)\left(k^{2}-2 k p^{\prime}+\Delta^{\prime}+i \epsilon\right)}\right|_{p=p^{\prime}} \\
& =\frac{2 i \kappa^{2} p^{4}}{16 \pi^{4}} \frac{1}{\left(k^{2}-2 k p+\Delta+i \epsilon\right)^{2}}
\end{aligned}
$$

where $\Delta=p^{2}-m^{2}, \Delta^{\prime}=p^{\prime 2}-m^{2}$ To see this, from Fig. (1), note that the Feynman rules give us the following result

$$
\begin{aligned}
i \Sigma_{1}(p)=\{ & -\frac{\int d^{4} k}{(2 \pi)^{4}} i v_{3}(p, p-k)_{\mu \bar{\mu}} \frac{i}{(p-k)^{2}-m^{2}+i \epsilon} i v_{3}\left(p-k, p^{\prime}\right)_{\nu \bar{\nu}} \\
& \frac{i \frac{1}{2}\left(\eta^{\mu \nu} \eta^{\bar{\mu} \bar{\nu}}+\eta^{\mu \bar{\nu}} \eta^{\bar{\mu} \nu}-\eta^{\mu \bar{\mu}} \eta^{\nu \bar{\nu}}\right)}{k^{2}-\lambda^{2}+i \epsilon} \\
& \left.-\frac{\int d^{4} k}{2(2 \pi)^{4}} i v_{4}\left(p, p^{\prime}\right)_{\mu \bar{\mu} ; j \bar{\nu}} \frac{i \frac{1}{2}\left(\eta^{\mu \nu} \eta^{\bar{\mu} \bar{\nu}}+\eta^{\mu \bar{\nu}} \eta^{\bar{\mu} \nu}-\eta^{\mu \bar{\mu}} \eta^{\nu \bar{\nu}}\right)}{k^{2}-\lambda^{2}+i \epsilon}\right\}\left.\right|_{p=p^{\prime}}
\end{aligned}
$$

where we have defined from the Feynman rules the respective3-point $(h \varphi \varphi)$ and 4-point $(h h \varphi \varphi)$ vertices

$$
\begin{gathered}
i v_{3}\left(p, p^{\prime}\right)_{\nu \bar{\nu}}=-i \kappa\left(p_{\nu} p_{\bar{\nu}}^{\prime}+p_{\bar{\nu}} p_{\nu}^{\prime}-g_{\nu \bar{\nu}}\left(p p^{\prime}-m^{2}\right)\right) \\
i v_{4}\left(p, p^{\prime}\right)_{\mu \bar{\mu} ; \nu \bar{\nu}}=-4 i \kappa^{2}\left[\left(p p^{\prime}-m^{2}\right)\left(\eta_{\mu \bar{\nu}} \eta_{\bar{\mu} \nu}+\eta_{\bar{\mu} \bar{\nu}} \eta_{\mu \nu}-\eta_{\mu \bar{\mu}} \eta_{\nu \bar{\nu}}\right)\right. \\
-\left(p^{\mu^{\prime}} p^{\prime \nu^{\prime}}+p^{\nu^{\prime}} p^{\prime \mu^{\prime}}\right)\left\{\eta_{\mu \mu^{\prime}}\left(\eta_{\bar{\mu} \nu} \eta_{\nu^{\prime} \bar{\nu}}+\eta_{\bar{\mu} \bar{\nu}} \eta_{\nu^{\prime} \nu}-\eta_{\nu^{\prime} \bar{\mu}} \eta_{\nu \bar{\nu}}\right)\right. \\
\left.\left.+\eta_{\bar{\mu} \mu^{\prime}}\left(\eta_{\mu \nu} \eta_{\nu^{\prime} \bar{\nu}}+\eta_{\mu \bar{\nu}} \eta_{\nu^{\prime} \nu}-\eta_{\nu^{\prime} \mu} \eta_{\nu \bar{\nu}}\right)\right\}\right]
\end{gathered}
$$

using the standard conventions so that $\mathrm{p}$ is incoming and $\mathrm{p}$ ' is outgoing for the scalar particle momenta at the respective vertices. In this way, we see that we may isolate the IR dominant part of $i \Sigma_{1}(p)$ by the separation

$$
\begin{aligned}
\frac{1}{k^{2}-2 k p+\Delta+i \epsilon}=- & \frac{\Delta}{\left(k^{2}-2 k p+\Delta+i \epsilon\right)^{2}}+\frac{1}{k^{2}-2 k p+i \epsilon} \\
& -\frac{2 \Delta^{2}}{\left(k^{2}-2 k p+\Delta+i \epsilon\right)^{2}\left(k^{2}-2 k p+i \epsilon\right)} \\
& -\frac{\Delta^{3}}{\left(k^{2}-2 k p+\Delta+i \epsilon\right)^{2}\left(k^{2}-2 k p+i \epsilon\right)^{2}} \\
& +\sum_{n=2}^{\infty}(-1)^{n} \frac{\Delta^{n}}{\left(k^{2}-2 k p+i \epsilon\right)^{n+1}}
\end{aligned}
$$

from which we can see that the first term on the RHS gives, upon insertion into (8), the IR-divergent contribution for the coefficient of the lowest order inverse propagator for the onshell limit $\Delta \rightarrow 0$. The second term does not produce an IRdivergence and the remaining terms vanish faster than $\Delta$ in the on-shell limit so that they do not contribute to the field renormalization factor which we seek to isolate. In this way we get finally

$$
\begin{aligned}
& i \Sigma_{1}(p)=\left\{-\frac{\int d^{4} k}{(2 \pi)^{4}}\left(-2 i \kappa p_{\mu} p_{\bar{\mu}}+i \delta v_{3}(p, p-k)_{\mu \bar{\mu}}\right)\left(\frac{-i \Delta}{\left(k^{2}-2 k p+\Delta+i \epsilon\right)^{2}}+i R \Delta_{F}(k, p)\right)\right. \\
&\left(-2 i \kappa p_{\nu}^{\prime} p_{\bar{\nu}}^{\prime}+i \delta v_{3}\left(p^{\prime}-k, p^{\prime}\right)_{\nu \bar{\nu}}\right) \frac{i \frac{1}{2}\left(\eta^{\mu \nu} \eta^{\bar{\mu} \bar{\nu}}+\eta^{\mu \bar{\nu}} \eta^{\bar{\mu} \nu}-\eta^{\mu \bar{\mu}} \eta^{\nu \bar{\nu}}\right)}{k^{2}-\lambda^{2}+i \epsilon} \\
&\left.-\frac{\int d^{4} k}{2(2 \pi)^{4}} i v_{4}\left(p, p^{\prime}\right)_{\mu \bar{\mu} ; \nu \bar{\nu}} \frac{i \frac{1}{2}\left(\eta^{\mu \nu} \eta^{\bar{\mu} \bar{\nu}}+\eta^{\mu \bar{\nu}} \eta^{\bar{\mu} \nu}-\eta^{\mu \bar{\mu}} \eta^{\nu \bar{\nu}}\right)}{k^{2}-\lambda^{2}+i \epsilon}\right\}\left.\right|_{p=p^{\prime}} \\
&=\left\{\frac { \int d ^ { 4 } k } { ( 2 \pi ) ^ { 4 } } \left[\left(-i \kappa p_{\bar{\mu}}\right)\left(2 i p_{\mu}\right) \frac{-i \Delta}{\left(k^{2}-2 k p+\Delta+i \epsilon\right)^{2}}\left(-i \kappa p_{\bar{\nu}}^{\prime}\right)\left(2 i p^{\prime}{ }_{\nu}\right)\right.\right. \\
&\left.\left.\frac{i \frac{1}{2}\left(\eta^{\mu \nu} \eta^{\bar{\mu} \bar{\nu}}+\eta^{\mu \bar{\nu}} \eta^{\bar{\mu} \nu}-\eta^{\mu \bar{\mu}} \eta^{\nu \bar{\nu}}\right)}{k^{2}-\lambda^{2}+i \epsilon}+\frac{(2 \pi)^{4} \beta_{1}(k)}{k^{2}-\lambda^{2}+i \epsilon}\right]\right\}\left.\right|_{p=p^{\prime}}
\end{aligned}
$$

which agrees with $(5,6,7)$ with

$$
\begin{aligned}
& R \Delta_{F}(k, p)=\frac{1}{k^{2}-2 k p+i \epsilon}-\frac{2 \Delta^{2}}{\left(k^{2}-2 k p+\Delta+i \epsilon\right)^{2}\left(k^{2}-2 k p+i \epsilon\right)} \\
& -\frac{\Delta^{3}}{\left(k^{2}-2 k p+\Delta+i \epsilon\right)^{2}\left(k^{2}-2 k p+i \epsilon\right)^{2}} \\
& +\sum^{\infty}(-1)^{n} \frac{\Delta^{n}}{\left(k^{2}-2 k p+i \epsilon\right)^{n+1}}, \\
& i \delta v_{3}(p, p-k)_{\mu \bar{\mu}}=i v_{3}(p, \bar{p}-k)_{\mu \bar{\mu}}-\left\{-2 i \kappa p_{\mu} p_{\bar{\mu}}\right\} \text {, } \\
& \beta_{1}(k)=\left\{-\frac{1}{(2 \pi)^{4}}\left(-2 i \kappa p_{\mu} p_{\bar{\mu}}+i \delta v_{3}(p, p-k)_{\mu \bar{\mu}}\right)\left[\frac{-i \Delta}{\left(k^{2}-2 k p+\Delta+i \epsilon\right)^{2}}\right.\right. \\
& \left.+i R \Delta_{F}(k, p)\right]\left(i \delta v_{3}\left(p^{\prime}-k, p^{\prime}\right)_{\nu \bar{\nu}}\right)\left\{i \frac{1}{2}\left(\eta^{\mu \nu} \eta^{\bar{\mu} \bar{\nu}}+\eta^{\mu \bar{\nu}} \eta^{\bar{\mu} \nu}-\eta^{\mu \bar{\mu}} \eta^{\nu \bar{\nu}}\right)\right\} \\
& -\frac{1}{(2 \pi)^{4}}\left(-2 i \kappa p_{\mu} p_{\bar{\mu}}+i \delta v_{3}(p, p-k)_{\mu \bar{\mu}}\right)\left(i R \Delta_{F}(k, p)\right) \\
& \left(-2 i \kappa p_{\nu}^{\prime} p_{\bar{\nu}}^{\prime}\right)\left\{i \frac{1}{2}\left(\eta^{\mu \nu} \eta^{\bar{\mu} \bar{\nu}}+\eta^{\mu \bar{\nu}} \eta^{\bar{\mu} \nu}-\eta^{\mu \bar{\mu}} \eta^{\nu \bar{\nu}}\right)\right\} \\
& -\frac{1}{(2 \pi)^{4}}\left(i \delta v_{3}(p, p-k)_{\mu \bar{\mu}}\right)\left(\frac{-i \Delta}{\left(k^{2}-2 k p+\Delta+i \epsilon\right)^{2}}\right) \\
& \left(-2 i \kappa p_{\nu}^{\prime} p_{\bar{\nu}}^{\prime}\right)\left\{i \frac{1}{2}\left(\eta^{\mu \nu} \eta^{\bar{\mu} \bar{\nu}}+\eta^{\mu \bar{\nu}} \eta^{\bar{\mu} \nu}-\eta^{\mu \bar{\mu}} \eta^{\nu \bar{\nu}}\right)\right\} \\
& \left.-\frac{1}{2(2 \pi)^{4}} i v_{4}\left(p, p^{\prime}\right)_{\mu \bar{\mu} ; \nu \bar{\nu}}\left\{i \frac{1}{2}\left(\eta^{\mu \nu} \eta^{\bar{\mu} \bar{\nu}}+\eta^{\mu \bar{\nu}} \eta^{\bar{\mu} \nu}-\eta^{\mu \bar{\mu}} \eta^{\nu \bar{\nu}}\right)\right\}\right\}\left.\right|_{p=p^{\prime}} .
\end{aligned}
$$

One can see that the result in (7) differs from the corresponding result in QED in eq. (5.13) of Ref. [33] by the replacement of the electron charges e by the gravity charges $\kappa p_{\bar{\mu}}, \kappa p_{\bar{v}}^{\prime}$ with the corresponding replacement of the photon propagator numerator $-i \eta_{\mu \nu}$ by the graviton propagator numerator $i \frac{1}{2}\left(\eta^{\mu v} \eta^{\bar{\mu} \bar{v}}+\eta^{\mu \bar{v}} \eta^{\bar{\mu} v}-\eta^{\mu \bar{\mu}} \eta^{\mu \bar{v}}\right)$. That the squared modulus of these gravity charges grows quadratically in the deep Euclidean regime is what makes their effect therein in the quantum theory of general relativity fundamentally different from the effect of the QED charges in the deep Euclidean regime of QED, where the latter charges are constants order-by-order in perturbation theory.

Indeed, proceeding recursively, we write

$\rho_{n}\left(k_{1}, \ldots, k_{n}\right)=S_{g}^{\prime \prime}\left(k_{n}\right) \rho_{n-1}\left(k_{1}, \ldots, k_{n-1}\right)+\beta_{n}^{(1)}\left(k_{1}, \ldots, k_{n-1} ; k_{n}\right)$

where here the notation indicates that the residual $\beta_{n}^{(1)}$ does not contain the leading infrared contribution for $k_{n}$ that is given by the first term on the RHS of (13). ${ }^{5 .}$ We iterate (13) to get

$$
\begin{aligned}
& \rho_{n}\left(k_{1}, \cdots, k_{n}\right)=S_{g}^{\prime \prime}\left(k_{n}\right) S_{g}^{\prime \prime}\left(k_{n-1}\right) \rho_{n-2}\left(k_{1}, \cdots, k_{n-2}\right) \\
& +S_{g}^{\prime \prime}\left(k_{n}\right) \beta_{n-1}^{(1)}\left(k_{1}, \cdots, k_{n-2} ; k_{n-1}\right) \\
& +S_{g}^{\prime \prime}\left(k_{n-1}\right) \beta_{n-1}^{(1)}\left(k_{1}, \cdots, k_{n-2} ; k_{n}\right) \\
& +\left\{-S_{g}^{\prime \prime}\left(k_{n-1}\right) \beta_{n-1}^{(1)}\left(k_{1}, \cdots, k_{n-2} ; k_{n}\right)+\beta_{n}^{(1)}\left(k_{1}, \cdots, k_{n-1} ; k_{n}\right)\right\}
\end{aligned}
$$

The symmetry of $\rho_{n}$ implies that the quantity in curly brackets is also symmetric in the interchange of $k_{n-1}$ and $k_{n}$. We indicate this explicitly with the notation

$$
\begin{aligned}
& \left\{-S_{g}^{\prime \prime}\left(k_{n-1}\right) \beta_{n-1}^{(1)}\left(k_{1}, \cdots, k_{n-2} ; k_{n}\right)+\beta_{n}^{(1)}\left(k_{1}, \cdots, k_{n-1} ; k_{n}\right)\right\} \\
& =\beta_{n}^{(2)}\left(k_{1}, \cdots, k_{n-2} ; k_{n-1}, k_{n}\right) .
\end{aligned}
$$

Repeated application of (13) and use of the symmetry of $\rho_{n}$ leads us finally to the exact result

${ }^{5} \mathrm{We}$ stress that it may contain in general other IR singular contributions. 
$\rho_{n}\left(k_{1}, \cdots, k_{n}\right)=S_{g}^{\prime \prime}\left(k_{1}\right) \cdots S_{g}^{\prime \prime}\left(k_{n}\right) \beta_{0}$

$+\sum_{i=1}^{n} S_{g}^{\prime \prime}\left(k_{1}\right) \cdots S_{g}^{\prime \prime}\left(k_{i-1}\right) S_{g}^{\prime \prime}\left(k_{i+1}\right) \cdots S_{g}^{\prime \prime}\left(k_{n}\right) \beta_{1}\left(k_{i}\right)$

$+\cdots+\sum_{i=1}^{n} S_{g}^{\prime \prime}\left(k_{i}\right) \beta_{n-1}\left(k_{1}, \cdots, k_{i-1}, k_{i+1}, \cdots, k_{n}\right)+\beta_{n}\left(k_{1}, \cdots, k_{n}\right)$

where the case $n=1$ has already been considered in (5) with $\rho_{0}$ $\equiv \beta_{0}$. Here, we defined as well $\beta_{i}^{(i)} \equiv \beta_{i}$.

We can use the symmetry of the residuals $\beta_{i}$ to re-write $\rho_{n}$ as $\rho_{n}\left(k_{1}, \cdots, k_{n}\right)=\sum_{p e r m} \sum_{r=0}^{n} \frac{1}{r !(n-r) !} \prod_{i=1}^{r} S_{g}^{\prime \prime}\left(k_{i}\right) \beta_{n-r}\left(k_{r+1}, \cdots, k_{n}\right)$

so that we finally obtain, upon substitution into (4),

$i \Sigma_{n}(p)=\sum_{r=0}^{n} \frac{1}{r !(n-r) !}\left(\int \frac{d^{4} k S_{g}^{\prime \prime}(k)}{k^{2}-\lambda^{2}+i \epsilon}\right)^{r} \int \prod_{i=1}^{n-r} \frac{d^{4} k_{i}}{k_{i}{ }^{2}-\lambda^{2}+i \epsilon} \beta_{n-r}\left(k_{1}, \cdots, k_{n-r}\right)$

With the definition

$-B_{g}^{\prime \prime}(p)=\int \frac{d^{4} k S_{g}^{\prime \prime}(k)}{k^{2}-\lambda^{2}+i \epsilon}$

and the identification

$$
i \Sigma_{r}^{\prime}(p)=\frac{1}{r !} \int \prod_{i=1}^{r} \frac{d^{4} k_{i}}{k_{i}^{2}-\lambda^{2}+i \epsilon} \beta_{r}\left(k_{1}, \cdots, k_{r}\right)
$$

we introduce the result (18) into (3) to get

$$
\begin{aligned}
-i\left(\Delta_{F}(p)^{-1}-\Sigma(p)\right) & =i \sum_{n=0}^{\infty} \sum_{r=0}^{n} \Sigma_{n-r}^{\prime}(p) \frac{\left(-B_{g}^{\prime \prime}(p)\right)^{r}}{r !} \\
& =i e^{-B_{g}^{\prime \prime}(p)} \sum_{\ell=0}^{\infty} \Sigma_{\ell}^{\prime}(p) \\
& =-i e^{-B_{g}^{\prime \prime}(p)}\left(\Delta_{F}(p)^{-1}-\sum_{\ell=1}^{\infty} \Sigma_{\ell}^{\prime}(p)\right) .
\end{aligned}
$$

In this way, our resummed exact result for the complete propagator in quantum general relativity is seen to be [8991]

$$
\left.i \Delta_{F}^{\prime}(p)\right|_{\text {resummed }}=\frac{i e^{B_{g}^{\prime \prime}(p)}}{\left(p^{2}-m^{2}-\Sigma_{s}^{\prime}(p)+i \epsilon\right)}
$$

where $^{6}$

$$
\Sigma_{s}^{\prime}(p) \equiv \sum_{\ell=1}^{\infty} \Sigma_{\ell}^{\prime}(p)
$$

Some observations are in order before we turn to the consequences of (22). First, we have not modified Einstein's theory at all. This means we are developing a very conservative approach to treat the UV behavior of of quantum

\footnotetext{
${ }^{6}$ To summarize what we have just done, note that (22) shows that $i \sum_{n=0}^{\infty} \sum_{n(p)}=-i e^{-} B_{g}^{\prime \prime(p)}\left(p^{2}-m^{2}+i \varepsilon-\sum_{\mathrm{s}}^{\prime}(p)\right)$, so that by elementary algebra we have the relation $-\Sigma_{n}(p)=-\sum_{j=0}^{n} \sum_{j}^{\prime}(p)\left(-B_{g}^{\prime \prime}(p)\right)^{n^{-j}} /(n-j)$ ! where $\sum_{j}^{\prime}(p)$ is the $\mathrm{j}$-th loop contribution to $\sum_{s}^{\prime}(p)$ with $-\sum_{0}^{\prime}(p)=$ $\Delta_{F}(p)^{-1}$ Every contribution to the exact Feynman series for $\Sigma(p)$ is reproduced exactly on the right-hand side of (22) -there is nothing missing and there is nothing extra.
}

general relativity. This makes our approach interesting in its own right, as we have noted in the Introduction. Second, because we did not modify the theory, what we have done is necessarily gauge invariant, as the original theory was gauge invariant. Third, the IR-improved $\sum_{s}^{\prime}(p)$ is already organized in a loop expansion by our derivation of (23). We expect therefore to be able to treat it perturbatively when the physics allows us to so do.

Let us stress that the resummation which we have done to derive (22) is valid for all $p$. When $p$ is in the deep UV one is resumming large IR effects due to the emission and reabsorption of IR gravitons by a particle with large Euclidean momenta. The effects are large because the momentum $p$ is large and the coupling of IR gravitons is proportional to $p$; the effects are therefore a property of the UV and they are in the UV. They are not extrapolated to the UV.

To see the effect of the exponential factoring (22), we evaluate the exponent as follows for Euclidean momenta (see Appendix 1 for the details of the attendant evaluation).

$B_{g}^{\prime \prime}(k)=\frac{\kappa^{2}\left|k^{2}\right|}{8 \pi^{2}} \ln \left(\frac{m^{2}}{m^{2}+\left|k^{2}\right|}\right)$.

The latter result establishes the advertised behavior: in the deep Euclidean regime, the resumed propagator falls faster than any finite power of $\left|k^{2}\right|$. This is exactly the type of behavior we need to tame the bad UV behavior of quantum general relativity. If $m=0$, we find, using the results in Appendix 1, that the standard factorization [107-111] of mass singularities allows us to isolate the large IR terms for $B_{g}^{\prime \prime}$ as

$\left.B_{g}^{\prime \prime}(k)\right|_{\text {factorized }}=\frac{\kappa^{2}\left|k^{2}\right|}{4 \pi^{2}} \ln \left(\frac{\left|\mu^{2}\right|}{\left|k^{2}\right|}\right)$

where $-\mu^{2}$ is a convenient Euclidean normalization point; the respective propagator again falls faster than any power of $\left|k^{2}\right|$ in the deep UV. We see as promised that the exponent $B_{g}^{\prime \prime}(k)$ has resumed in (24) terms $\mathcal{O}\left(G_{N}\left|k^{2}\right| \ln \left|k^{2}\right|\right)^{n,} n=0, \ldots, \infty$ for $k^{2}$ $\rightarrow \infty$. The use of the results in Refs. [112-114] by Refs. [10$12]$ is similar to what we do here. ${ }^{8}$

${ }^{7}$ See also Ref. [33], where this result can be inferred fromits eq. (5.17) by the substitution $\mathrm{e}^{2} \rightarrow-\kappa^{2} \mathrm{p}^{2}$ therein, as we have indicated above, where $\mathrm{p} \equiv \mathrm{k}$ here.

${ }^{8}$ Notice that, while the quantity e ${ }^{B_{g}^{\prime \prime}(p)}$ if expanded out order by order in powers of $B_{g}^{\prime \prime}(p)$, would diverge in each such order as $\left|k^{2}\right| \rightarrow \infty$, upon summing each such term, we get exponential damping, just as in QCD [1012], where, working to one-loop in the beta function $\beta(g)$ [112-114], by which we have the effective coupling evolution $d \bar{g}(t) / d t=\beta(\bar{g}(t))$, we have the running QCD coupling (here $g_{0}$ is the initial value at the reference scale, $t$ is the logarithm of the ratio of the observed scale to that reference scale and $\beta(g) \equiv-b_{0} g^{3}+\ldots$, with $\left.b_{0}>0\right) \bar{g}(t)^{2}=\frac{g_{0}^{2}}{1+2 b_{0} g_{0}^{2} t}$ so that, even though

each term $g_{0}^{2}\left(-2 b_{0} g_{0}^{2} t\right)^{n}, \quad n>0$, from the respective perturbation series, $g_{0}^{2}\left(1+\left(-2 b_{0} g_{0}^{2} t\right)+\left(-2 b_{0} g_{0}^{2} t\right)^{2}+\cdots\right)$, diverges as $t$ goes to $\infty$, the sum gives us the result for $\bar{g}(t)$ that vanishes as $t \rightarrow \infty$. Experiment has verified that expanding QCD in $\bar{g}(t)$ agrees with data for very large values of $t$ as we noted in the Introduction. The validity of the renormalization group equation for all values of $t$ means we can resum the series in $\left(-2 b_{0} g_{0}^{2} t\right)$ without requiring that $\left(-2 b_{0} g_{0}^{2} t\right)$ is small compared to 1 ; the validity of (22) for all values of $\mathrm{p}$ means that the resummed powers of $B_{g}^{\prime \prime}(p)$ do not have any restriction that $\left|p^{2}\right| G_{N}$ be small compared 1 . 
We have in fact shown in Ref. [89] that the exponentially damped behavior in the the propagator in (22), which holds for all particles because gravity couples in the infrared universally to all particles, leads to the superficial UV finiteness of quantum general relativity, which is completely consistent with asymptotic safety [68]. The proof is given explicitly in Ref. [89] -see especially pages 6-8 of the latter reference - for completeness, we record it in Appendix 3 here. In the next section, we turn to some of the further consequences of the improved propagator behavior and superficial UV finiteness we have found in our new approach to quantum general relativity.

\section{RESUMMED QUANTUM GRAVITY NEWTON'S LAW: SOME CONSEQUENCES}

AND

An immediate consequence of our new UV finite quantum loop results for QGR is that we can make exact, UV finite, predictions for the quantum loop corrections [8997] to Newton's law. These results are then unique because we do not modify Einstein's theory or quantum mechanics to obtain them and we have no free parameters. We now present our prediction for the quantum loop corrections to Newton's law in this Section.

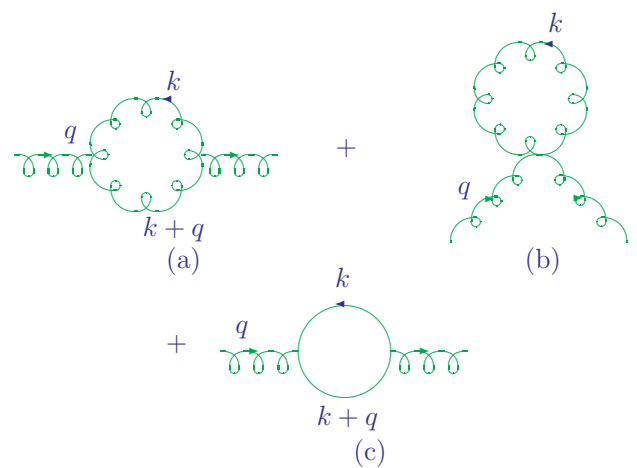

Fig. (2). The graviton ((a), (b)) and its ghost ((c)) one-loop contributions to the graviton propagator. $q$ is the 4-momentum of the graviton.

Specifically, consider the diagrams in Figs. (2) and (3). These graphs have a superficial degree of divergence in the UV of +4 and in the usual treatment of the theory they are well-known to generate a UV divergence in the respective 1PI2-point function for the coefficient of $q^{4}$, a divergence that thus can not be removed by the standard field and mass renormalizations. Any successful treatment of the UV behavior of QGR must therefore render this divergence finite. Indeed, when the graphs Figs. (2) and (3) are computed in our resummed quantum gravity theory, this is precisely what happens.

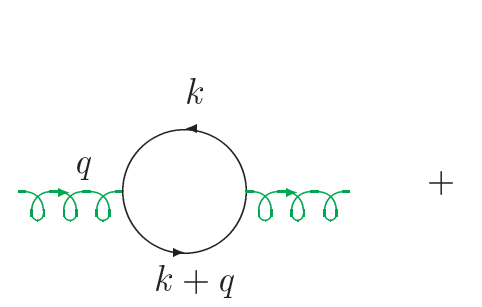

(a)

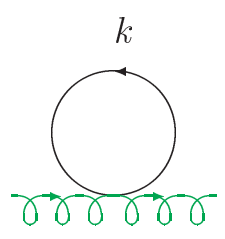

(b)
Fig. (3). The scalar one-loop contribution to the graviton propagator. $q$ is the 4-momentum of the graviton.
For example, consider the graph in Fig. (3a). When we use our resumed propagators, we get (here, $k \rightarrow\left(i k^{0}, \vec{k}\right)$ by Wick rotation, and we work in the transverse-traceless space).

$$
\begin{aligned}
i \Sigma(q)_{\bar{\mu}}^{1 a} ; \mu \nu & =i \kappa^{2} \frac{\int d^{4} k}{2(2 \pi)^{4}} \frac{\left(k_{\bar{\mu}}^{\prime} k_{\bar{\nu}}+k_{\bar{\nu}}^{\prime} k_{\bar{\mu}}\right) e^{\frac{\kappa^{2}\left|k^{\prime 2}\right|}{8 \pi^{2}} \ln \left(\frac{m^{2}}{m^{2}+\left|k^{\prime 2}\right|}\right)}}{\left(k^{\prime 2}-m^{2}+i \epsilon\right)} \\
& \frac{\left(k_{\mu}^{\prime} k_{\nu}+k_{\nu}^{\prime} k_{\mu}\right) e^{\frac{\kappa^{2}\left|k^{2}\right|}{8 \pi^{2}} \ln \left(\frac{m^{2}}{m^{2}+\left|k^{2}\right|}\right)}}{\left(k^{2}-m^{2}+i \epsilon\right)} .
\end{aligned}
$$

We see explicitly that the exponential damping in the deep Euclidean regime has rendered the graph in Fig. (3a) finite in the UV. Similarly are all the graphs in Figs. (2) and (3) UV finite when we use our respective resummed propagators to compute them.

To evaluate the effect of the corrections in Figs. (2) and (3) on the graviton propagator, we continue to work in the transverse, traceless space and isolate the effects from Figs. (2) and (3) on the coefficient of the $\mathrm{q}^{4}$ in the graviton propagator denominator,

$q^{2}+\frac{1}{2} q^{4} \Sigma^{T(2)}+i \epsilon$

so that we need to evaluate the transverse, traceless selfenergy function $\Sigma^{T}\left(q^{2}\right)$ that follows from eq. (26) for Fig. (3a) and its analogs for Figs. (3b) and (2) by the standard methods. Here, we work in the expectation that, in consequence to the newly UV finite calculated quantum loop effects in Figs. (2) and (3), the Fourier transform of the graviton propagator that enters Newton's law, our ultimate goal here, will receive support from from $|q|^{2}<<M_{p l}^{2}$. We will therefore work in the limit that $q^{2} / M_{p l}^{2}$ is relatively small, $\lesssim .1$, for example. ${ }^{9}$ This will allow us to see the dominant effects of our new finite quantum loop effects. In other words, we will work to $\sim 10 \%$ (leading-log) accuracy in what follows. See Appendix 2 for more discussion on this point.

First let us dispense with the contributions from Figs. (2b) and Fig. (3b). These are independent of $q^{2}$ so that we use a mass counter-term to remove them and set the graviton mass to 0. Following the suggestion of Feynman in Ref. [23], we will change this to a small non-zero value below to take into account the recently established small value of the cosmological constant [101]. See also the discussion in Ref. [115-118] where it is shown that the quantum fluctuations in the exact de Sitter metric implied by the non-zero cosmological constant correspond in general to a mass for the graviton. Here, as we expand about a flat background, we take this effect into account as a small infrared regulator for the graviton. The deviations from flat space in the deep Euclidean region that we study due to the observed value of the cosmological constant are at the level of $e^{10-61}-1$ ! This is safely well beyond the accuracy of our methods.

\footnotetext{
${ }^{9}$ This regime is for numerical convenience only, as it allows us to work with a simple quadratic equation in $q$ in determining the Fourier transform of the graviton propagator below. It is justified because the pole position which we find at non-zero $q^{2}$ satisfies it. There is no problem of principle to treat the exact result, and it will appear elsewhere.
} 
Returning to Fig. (3a), when we project onto the transverse, traceless space, that is to say, the graviton helicity space $\left\{e^{\mu v}( \pm 2)=\varepsilon_{ \pm}^{\mu} \varepsilon_{ \pm}^{v}\right.$, where $\varepsilon_{ \pm}^{v}= \pm(\hat{x} \pm i \hat{y}) / \sqrt{2}$ when $\hat{x}, \hat{y}$ are purely space-like and $(\vec{x}, \vec{y}, \vec{q} /|\vec{q}|)$ form a righthanded coordinate basis $\}$, we get (see the Appendix 2) the result

$i \Sigma^{T}\left(q^{2}\right)_{3 a}=\frac{-i \kappa^{2} m^{4}}{96 \pi^{2}} \int_{0}^{1} d \alpha \int_{0}^{\infty} d x \frac{x^{3}\left(2(x+1) \bar{d}+\bar{d}^{2}\right)}{(x+1)^{2}(x+1+\bar{d})^{2}}(1+x)^{-\lambda_{c} x}$

where $\lambda_{\mathrm{c}}=\frac{2 m^{2}}{\pi M_{p l}^{2}}, \bar{d}=\alpha(1-\alpha) \vec{q}^{2} / m^{2}$ so that we have made the substitution $x=k^{2}$ and imposed the mass counter-term as we noted. We have taken for definiteness $q=(0, \vec{q})$. We also use $q=|\vec{q}|$ when there is no chance for confusion. We are evaluating (28) in the deep UV where $\mathrm{m}^{2} / q^{2}<<1$ and where $q^{2} / M_{p l}^{2} \lesssim .1$ - see footnote 9 . Accordingly, we get

$i \Sigma^{T}\left(q^{2}\right)_{3 a}=\frac{-i \kappa^{2}}{96 \pi^{2}}\left(\frac{|\vec{q}|^{2} m^{2} c_{1}}{3}+\frac{|\vec{q}|^{4} c_{2}}{30}\right)$

where

$$
\begin{aligned}
& c_{1}=I_{1}\left(\lambda_{c}\right)=\int_{0}^{\infty} d x x^{3}(1+x)^{-3-\lambda_{c} x} \\
& c_{2}=I_{2}\left(\lambda_{c}\right)=\int_{0}^{\infty} d x x^{3}(1+x)^{-4-\lambda_{c} x} .
\end{aligned}
$$

Using the usual field renormalization, we see that Fig. (3a) makes the contribution

$$
i \tilde{\Sigma}^{T}\left(q^{2}\right)_{3 a} \cong \frac{-i \kappa^{2}|\vec{q}|^{4} c_{2}}{2880 \pi^{2}}
$$

to the transverse traceless graviton proper self-energy function.

Turning now to Fig. (2), the pure gravity loops, we use a contact between our work and that of Refs. [119]. In Refs. [119], the entire set of one-loop divergences have been computed for the theory in (2). The basic observation is the following. As we work only to the leading logarithmic accuracy in $\ln \lambda_{c}$, it is sufficient to identify the correspondence between the divergences as calculated in the n-dimensional regularization scheme in Ref. [119] and as they would occur when $\lambda_{c} \rightarrow 0$. This we do by comparing our result for (28) when $q^{2} \rightarrow 0$ with the corresponding result in Ref. [119] for the same theory. In this way we see that we have the correspondence

$-\ln \lambda_{c} \leftrightarrow \frac{1}{2-n / 2}$.

This allows us to read-off the leading log result for the pure gravity loops directly from the results in Ref. [119]. Since $-\ln \lambda_{c}=\ln M_{P l}^{2}-\ln m^{2}-\ln \frac{2}{\pi}$, we see that our exponentiated propagators have cut-off our UV divergences at the scale $\sim M_{P l}$ and the correspondence in (32) shows the usual relation between the effective UV cut-off scale and the pole in $(2-n / 2)$ in dimensional regularization. Note as well that, if the small cosmological constant [101] is set to zero ${ }^{10,}$ the graviton is then exactly mass less and we normalize its propagator at a Euclidean point $p^{2}=-\mu^{2}$ as is standard for massless non-Abelian gauge theories for example. It follows that for the graviton case and for all other cases where $m=0$, as we note in (25) and as we explain in Appendix 1 (see eq. (49)), the mass $m$ in (32) is replaced with $m=\mu$ - there is no zero mass divergence in the case that the mass of the respective particle is zero. The UV correspondence is the same in both the $m \neq 0$ and $m=0$ cases.

Specifically, the result in Ref. [119], when interpreted as we have just explained, is that the pure gravity loops give a factor of 42 times the scalar loops for the coefficient $a_{2}$ above when we work in the regime where $\left|q^{2}\right|$ is relatively small compared to $M_{p l}^{2}$. Here, we again take into account the recent significant evidence for a non-zero cosmological constant [101], which can be seen to provide the small nonzero rest mass for the graviton, $m_{g} \cong 3.1 \times 10^{-33} \mathrm{eV}$, which serves as an IR regulator for the graviton. This is the value of rest mass in $\lambda_{c}$ which should be used for pure gravitational loops - see footnote 10 for more discussion on this point relevant to Refs. [120, 121]. See the Appendix 1 for the derivation of the corresponding infrared exponents.

We note that, for $\lambda_{c}=0$, the constant $c_{2}$ is infinite and, as we have already imposed both the mass and field renormalization counter-terms, there would be no physical parameter in to which that infinity could be absorbed: this is just another manifestation that QGR, without our resummation, is a non-renormalizable theory.

Using the universality of the coupling of the graviton when the momentum transfer scale is relatively small compared to $M_{P l}$, we can extend the result for the scalar field above to the remaining known particles in the Standard Model by counting the number of physical degrees of freedom for each such particle and replacing the mass of the scalar with the respective mass of that particle. For a massive fermion we get a factor of 4 relative to the scalar result with the appropriate change in the mass parameter from $m$ to $m_{f}$, the mass of that fermion, for a massive vector, we get a factor

\footnotetext{
${ }^{10}$ Forthe reader unfamiliar with Feynman's original observation [23] that, in his approach to QGR, one of the main effects of the cosmological constant is to give the quantum graviton field $h_{\mu v}$ a mass, we recall Einstein's equation $R_{\mu v}-\frac{1}{2} g_{\mu v} R+\Lambda g_{\mu v}=-\kappa^{2} T_{\mu v}$, with $R_{\mu v}$ and $T_{\mu v}$ the respective Ricci and energy-momentum tensors. For $g_{\mu v}=\eta_{\mu v}+2 \kappa h_{\mu v}$, we get $R_{\mu v}=\kappa r_{\mu v}+$ $\mathcal{O}\left(\kappa^{2}\right)$, with $r_{\mu v}=h_{\mu v}-\partial_{\alpha} \partial_{\mu} h_{v}^{\alpha}+\partial_{\alpha} \partial_{v} h_{\mu}^{\alpha}+\partial_{\mu} \partial_{v} h_{\alpha}^{\alpha}$ so that, absorbing the $\Lambda \eta_{\mu v}$ term into the normal ordering constant $\eta_{\mu v}$ term in $T_{\mu v}$, we get the result $r_{\mu v}-\frac{1}{2} \eta_{\mu v} r+2 \Lambda h_{\mu v}=\kappa T_{\mu v}^{\prime}$ where here $T_{\mu v}^{\prime}$ is now the normal ordered energy-momentum tensor, including the contribution from the graviton itself. This result shows that the field $h_{\mu v}$, as already noted by Feynman [23], now has mass-squared $2 \Lambda$ working to leading order in $\Lambda$. We treat this as an IR regulator mass for a massless spin 2 field in Minkowski space over the Planck scale distances with which we work. Indeed, the nonzero value of $\Lambda$ means the background metric should be of de Sitter type and this avoids the problems noted in Refs. $[120,121]$ associated with a graviton mass different from zero in Minkowski space, as we explained further in the text above.
} 
of 3 relative to the scalar result, with the corresponding change in the mass from $\mathrm{m}$ to $m_{V}$, the mass of that vector, etc. In this way, we arrive at the result that the denominator of the graviton propagator becomes, in the Standard Model,

$q^{2}+\Sigma^{T}\left(q^{2}\right)+i \epsilon \cong q^{2}-q^{4} \frac{c_{2, e f f}}{360 \pi M_{P l}^{2}}$,

where we have defined

$$
\begin{aligned}
c_{2, \text { eff }} & =\sum_{\text {SM particles j }} n_{j} I_{2}\left(\lambda_{c}(j)\right) \\
& \cong 2.56 \times 10^{4}
\end{aligned}
$$

with $I_{2}$ defined above and with $\lambda_{c}(j)=\frac{2 m^{2}}{\pi M_{p l}^{2}}$ and [91] $n_{j}$ equal to the number of effective degrees of particle $j$ as already illustrated. In arriving at (34), we take the SM masses as follows: for the now presumed three massive neutrinos [122, 123], we estimate a mass at $\sim 3 \mathrm{eV}$; for the remaining members of the known three generations of Dirac fermions $\{e, \mu, \tau, u, d, s, c, b, t\}$, we use [124-126] $m_{e}$ $\cong 0.51 \mathrm{MeV}, m_{\mu} \cong 0.106 \mathrm{GeV}, m_{\tau} \cong 1.78 \mathrm{GeV}, m_{u} \cong 5.1$ $\mathrm{MeV}, m_{d} \cong 8.9 \mathrm{MeV}, m_{s} \cong 0.17 \mathrm{GeV}, m_{c} \cong 1.3 \mathrm{GeV}, m_{b} \cong 4.5$ $\mathrm{GeV}$ and $m_{t} \cong 174 \mathrm{GeV}$ and for the massive vector bosons $W^{ \pm}, Z$ we use the masses $M_{W} \cong 80.4 \mathrm{GeV}, M_{Z} \cong 91.19 \mathrm{GeV}$, respectively. We note that (see the Appendix1) when the rest mass of particle $j$ is zero, such as it is for the photon and the gluon, the value of $m_{j}$ turns-out to be $\sqrt{2}$ times the gravitational infrared cut-off mass [101], which is $m_{g}=$ $\cong 3.1 \times 10^{-33} \mathrm{eV}$.We further note that, from the exact one-loop analysis of Ref. [119], it also follows that the value of $n_{j}$ for the graviton and its attendant ghost is 42 . For $\lambda_{c} \rightarrow 0$, we have found the approximate representation

$I_{2}\left(\lambda_{c}\right) \cong \ln \frac{1}{\lambda_{c}}-\ln \ln \frac{1}{\lambda_{c}}-\frac{\ln \ln \frac{1}{\lambda_{c}}}{\ln \frac{1}{\lambda_{c}}-\ln \ln \frac{1}{\lambda_{c}}}-\frac{11}{6}$.

If we use the standard Fourier transform of the respective graviton propagator we obtain the improved Newton potential

$\Phi_{N}(r)=-\frac{G_{N} M}{r}\left(1-e^{-a r}\right)$,

where with

$a \cong\left(\frac{360 \pi M_{P l}^{2}}{c_{2, e f f}}\right)^{\frac{1}{2}}$

we have that

$a \cong 0.210 M_{P l}$

We note that the implied behavior of the running Newton constant, $G_{N}(k)$, that corresponds ${ }^{11}$ to our resummed graviton propagator denominator,

\footnotetext{
${ }^{11}$ We recall for the reader the Ward-Takahashi identities for the 1PI scalarscalar-graviton3pt function $\Gamma^{\mu v}$ which follow from the conservation of $T^{\mu v}$ : if the matter incoming 4-momentum is $\ell$, the matter outgoing 4-momentum is $\mathrm{k}$, and the outgoing graviton 4-momentum is $p=\ell-k$, the conservation law $\partial_{\mu} T^{\mu v}=0$ implies the Ward-Takahashi identities $(\ell-k)_{\mu} \Gamma^{\mu v}(p, \ell, k)=$ $-\ell^{v} \Delta_{F}^{\prime}{ }^{-1}(k)+k^{v} \Delta_{F}^{\prime}(\ell)^{-1}$, so that the matter field renormalization constant $Z_{2}$ exactly cancels the vertex renormalization constant $Z_{1}$ - this leaves the graviton vacuum polarization as the source of the running of Newton's constant, by the standard arguments. Here, $\Delta_{F}^{\prime}(\ell)$ is the complete scalar $2 \mathrm{pt}$ function.
}

$G_{N}(k)=\frac{G_{N}}{1+k^{2} / a^{2}}$

agrees with the large (Euclidean) $k^{2}$ limit of $G_{N}(k)$ found by the authors in Ref. [71] using the asymptotic safety approach $^{12}$ as realized by phenomenological exact renormalization group methods -we agree as well on the generic size of $a$. The connection between $\mathrm{k}$ and position space in our analysis is given by the usual Fourier trans formation method whereas that in Ref. [71] involves a phenomenological parameter which is ideally determined self-consistently. Thus, as we will see below, while our results and the results in Ref. [71] agree on $G_{N}(k)$ for large values of $k$, our forms for the corresponding Newton potential differ in position space: we expect our result to hold in the deep Euclidean regime whereas at larger distances the result in Ref. [71] should be preferred.

We also note that the behavior of the graviton propagator found by our analysis and by that in Ref. [71] agrees for large Euclidean $k^{2}$ with that in the $R^{2}$-quantum gravity theory $[127,128]$. However, unlike the latter theory, in our work unitarity has not been lost, as we quantize the theory using the methods of Refs. [6-9, 22, 23].

We discuss now two consequences of the improved Newton potential:

\subsection{Elementary Particles and Black Holes}

One of the issues that confronts the theory of point particle fields is that fact that a massive point particle of rest mass $\mathrm{m}$ has its mass entirely inside of its Schwarzschild radius $\mathrm{r}_{\mathrm{S}}=2 \mathrm{~m} / M_{p l}^{2}$ so that classically it should be a black hole. We expect this conclusion to be modified by quantum mechanics, where the mass of such a particle seems readily accessible in experiments. Note that we distinguish here the uncertainty in the position of the particle, which is connected to its Compton wavelength when the particle is at rest, from the accessibility of the mass of that particle, which is connected to its black hole character. The situation can be addressed by focusing on the lapse function in the metric class

$d s^{2}=f(r) d t^{2}-f(r)^{-1} d r^{2}-r^{2} d \Omega^{2}$,

with

$f(r)=1-\frac{2 G(r) m}{r}$

and $G(r)$, using (36), given by

$G(r)=G_{N}\left(1-e^{-a r}\right)$.

We see that the Standard Model massive particles all have the property that $f(r)$ remains positive as $r$ passes through their respective Schwarzschild radii and goes to $r=$ 0 , so that the particle is no longer $[90,91]$ a black hole as it was classically. Refs. [71, 128] have also found that subPlanck mass black holes do not exist in quantum field theory.

\footnotetext{
${ }^{12}$ Our UV fixed-point behavior of the dimensionless coupling $k^{2} G_{N}(k)$ is the four-dimensional version of the fixed point found by Weinberg [68] in $2+\varepsilon$ dimensions; see Ref. [71] for more discussion of this point as well.
} 


\subsection{Final State of Hawking Radiation - Planck Scale Cosmic Rays}

The situation that then naturally comes to mind is the evaporation of massive black holes. In Ref. [71], following Weinberg's [68] asymptotic safety approach as realized by phenomenological exact renormalization group methods, it has been shown that the attendant running of Newton's constant $[130]^{13}$ leads to the lapse function representation, in the metric class in (40)

$f(r)=1-\frac{2 G(r) M}{r}$

where $M$ is the mass of the black hole and now

$G(r) \equiv G_{B R}(r)=\frac{G_{N} r^{3}}{r^{3}+\tilde{\omega} G_{N}\left[r+\gamma G_{N} M\right]}$

where $\gamma$ is a phenomenological parameter [71] satisfying $0 \leq$ $\gamma \leq \frac{9}{2}$ and $\tilde{\omega}=\frac{118}{15 \pi}$. It can be shown that (44) leads as well to the conclusion that black holes with mass less than a critical mass $M_{c r} \sim M_{P l}$ have no horizon, as we have argued for massive SM elementary particles. When we join our resulting (41) on to that in (44) at the outer most solution, $r$, of the equation

$$
G_{B R}(r)=G_{N}\left(1-e^{-a r}\right),
$$

we have a result for the final state of the Hawking process for an originally very massive black hole: for $r\langle r\rangle$, in the lapse function we use our result in (41) for $G(r)$ and for $r>$ $r>$ we use $G_{B R}(r)$ for $G(r)$ after the originally massive black hole has Hawking radiated down to the appropriate scale. For example, for the self-consistent value $\gamma=0$ and $0.2=\Omega$ $\equiv \frac{\tilde{\omega}}{G_{N} M^{2}}=\frac{\tilde{\omega} M_{p l}^{2}}{M^{2}}$ for definiteness we find [92-97] that the inner horizon found in Ref. [71] moves to negative values of $r$ and that the outer horizon moves to $r=0$, so that the entire mass of the originally very massive black hole radiates away until a Planck scale remnant of mass $M_{c r}^{\prime}=2.38 M_{P l}$ is left [71, 131-133], which then is completely accessible to our universe. It would be expected to decay into n-body final states, $n=2,3, \ldots$, leading in general to Planck scale cosmic rays [91-97]. The data in Ref. [134-141] are not inconsistent with this conclusion, which also agrees with recent results by Hawking [142].

\section{CONCLUSIONS}

In this paper we have introduced a superficially UV finite theory of the quantum general relativity. This is achieved by resumming the large IR terms $\mathcal{O}\left(G_{N}\left|k^{2}\right| \ln \left|k^{2}\right|\right)^{n} n \geq 0$ in the deep UV regime $\left|k^{2}\right| \rightarrow \infty$ and hence the reader has been cautioned of the strict mathematical convergence issues that are beyond the scope of our discussion ${ }^{14}$. This latter caution notwithstanding, our approach would appear to be a solution to most of the outstanding problems in the union of the ideas

\footnotetext{
${ }^{13}$ See Ref. [130] for a discussion of the gauge in variance issues here.
}

of Bohr and Einstein. More importantly, it makes a strong argument that quantum mechanics, while not necessarily the ultimate theory, is not an incomplete theory.

Our approach to quantum gravity does not contradict any known experimental or theoretical fact; rather, it allows us to better understand the known physics and, hopefully, to make new testable predictions. Our approach does not contradict string theory or loop quantum gravity, to the best of our knowledge. In principle, all three approaches to quantum general relativity should agree in the appropriate regimes, where we would stress that, unlike what is suggested by the other two approaches, sub-Planck scale phenomena do exist in our approach. Further work on establishing the precise relationship between the three approaches is in progress.

Evidently, formulations for supergravity theories in Refs. $[69,143-145]$ which were abandoned as complete theories of quantum gravity because they proved to be non renormalizable are now, with the resummation methods of this paper, rendered superficially UV finite in the sense defined in the Introduction and thus are again phenomenologically interesting in their own right rather than as low energy approximations to surper-string theory. Of course, they may still have other problems. We will pursue this line of phenomenology elsewhere.

\section{ADDED NOTE}

Recently, D. Kreimer [146] has shown, at least for the pure gravity part of Einstein's theory, using the Hopfalgebraic Dyson-Schwinger equation realization of renormalization theory [147], that, while quantum gravity is non-renormalizable order by order in perturbation theory, there is an infinite set of relations among residues of the respective amplitudes so that when all are imposed only a finite number of unknown constants obtain, i.e., he finds in this way more evidence that quantum gravity is nonperturbatively renormalizable. This finding is entirely consistent with the results in Refs. [71-74] as well as with our results presented herein. We find here in addition that the quantum general relativity is in fact superficially UV finite and that, as argued in Refs. [71-74], it has asymptotically safe UV fixed point behavior. Presumably, when the complete analysis is done using the methods in Ref. [146], the latter behavior will also be recovered.

\footnotetext{
${ }^{14}$ That the structure of gravity is essential to the UV finiteness is due to the proportionality to the 4 momentum of its IR coupling for gravitons to all elementary particles, as we have explained: for example, in the SM, the $Z^{0}$ and neutrinos have no couplings to photons, so even if we YFS resum all propagators for large photonic and gluonic IR effects, we do not change the $\mathrm{UV}$ behavior for the $\mathrm{Z}^{0}$ and neutrino propagators, leaving neutrino and $Z^{0}$ self-energies still UV divergent. If we resum the large gravitational IR effects, these self-energies are also rendered finite. If a dimension six New Physics contact interaction is added to the SM, the marginal UV improvement from resumming the large IR photonic and gluonic effects still leaves the loops with this contact interaction UV divergent; including the gravitational IR resummation renders the loops finite. Of course, the key to this finiteness is the structure of the YFS theory which allows the divergent effective coupling $\left|k^{2}\right| G_{N}$ to generate exponential suppression faster than any power of $\left|k^{2}\right|$ for $\left|k^{2}\right| \rightarrow \infty$ in the deep UV - this is one of our main points in our discussion.
} 


\section{ACKNOWLEDGEMENTS}

We thank Profs. S. Bethke and L. Stodolsky for the support and kind hospitality of the MPI, Munich, while a part of this work was completed. We thank Prof. S. Jadach for useful discussions. This work is partly supported by the US Department of Energy grant DE-FG02-05ER41399 and by NATO Grant PST.CLG.980342.

\section{APPENDIX 1: EVALUATION OF GRAVITATIONAL INFRARED EXPONENT}

In the text, we use several limits of the gravitational infrared exponent $B_{g}^{\prime \prime}$ defined in (19). Here, we present these evaluations for completeness.

We have to consider

$$
\begin{aligned}
& -B_{g}^{\prime \prime}(p)=\int \frac{d^{4} k S_{g}^{\prime \prime}(k)}{k^{2}-\lambda^{2}+i \epsilon} \\
& =\left.\int \frac{d^{4} k}{\left(2 \pi \pi^{4}\left(k^{2}-\lambda^{2}+i \epsilon\right)\right.} \frac{i \frac{1}{2}\left(\eta^{\mu \nu} \eta^{\bar{\mu} \bar{\omega}}+\eta^{\mu \bar{\nu}} \eta^{\bar{\mu} \nu}-\eta^{\mu \bar{\mu}} \eta^{\nu \bar{\nu}}\right)\left(-i \kappa p_{\bar{\mu}}\right)\left(2 i p_{\mu}\right)\left(-i \kappa p_{\bar{\nu}}^{\prime}\right)\left(2 i p_{\nu}^{\prime}\right)}{\left(k^{2}-2 k p+\Delta+i \epsilon\right)\left(k^{2}-2 k p^{\prime}+\Delta^{\prime}+i \epsilon\right)}\right|_{p=p^{\prime}} \\
& =\frac{2 i \kappa^{2} p^{4}}{16 \pi^{4}} \int \frac{d^{4} k}{\left(k^{2}-\lambda^{2}+i \epsilon\right)} \frac{1}{\left(k^{2}-2 k p+\Delta+i \epsilon\right)^{2}}
\end{aligned}
$$

where $\Delta=p^{2}-m^{2}$. The integral on the RHS of (46) is given by

$$
\begin{aligned}
I & =\int \frac{d^{4} k}{\left(k^{2}-\lambda^{2}+i \epsilon\right)} \frac{1}{\left(k^{2}-2 k p+\Delta+i \epsilon\right)^{2}} \\
& =\frac{-i \pi^{2}}{p^{2}} \frac{1}{x_{+}-x_{-}}\left[x_{+} \ln \left(1-1 /\left(\sqrt{2} x_{+}\right)\right)-x_{-} \ln \left(1-1 /\left(\sqrt{2} x_{-}\right)\right)\right]
\end{aligned}
$$

with

$x_{ \pm}=\frac{1}{2 \sqrt{2}}\left(\bar{\Delta}+\bar{\lambda}^{2} \pm\left(\left(\bar{\Delta}+\bar{\lambda}^{2}\right)^{2}-4\left(\bar{\lambda}^{2}-i \bar{\epsilon}\right)\right)^{1 / 2}\right)$

for $\bar{\Delta}=1-m^{2} / p^{2}, \bar{\lambda}^{2}=\lambda^{2} / p^{2}$ and $\bar{\varepsilon}=\varepsilon / p^{2}$. In this way, we arrive at the results, for $p^{2}<0$,

$$
B_{g}^{\prime \prime}(p)= \begin{cases}\frac{\kappa^{2}\left|p^{2}\right|}{8 \pi^{2}} \ln \left(\frac{m^{2}}{m^{2}+\left|p^{2}\right|}\right), & m \neq 0 \\ \frac{\kappa^{2}\left|p^{2}\right|}{8 \pi^{2}} \ln \left(\frac{m_{g}^{2}}{m_{g}^{2}+\left|p^{2}\right|}\right), & m=m_{g}=\lambda \\ \frac{2 \kappa^{2}\left|p^{2}\right|}{8 \pi^{2}} \ln \left(\frac{m_{g}^{2}}{\left|p^{2}\right|}\right), & m=0, m_{g}=\lambda\end{cases}
$$

where we have made more explicit the presence of the observed small mass, $m_{g}$, of the graviton. When $\mathrm{m}=0$ and one wants to use dimensional regularization for the IR regime instead of $m_{g}$, we normalize the propagator at a Euclidean point $k^{2}=-\mu^{2}$ and use standard factorization arguments [107-111] to take the factorized result for $B_{g}^{\prime \prime}$ from (48) as

$\left.B_{g}^{\prime \prime}(p)\right|_{\text {factorized }}=\frac{2 \kappa^{2}\left|p^{2}\right|}{8 \pi^{2}} \ln \left(\frac{\left|\mu^{2}\right|}{\left|p^{2}\right|}\right), \quad m=0, m_{g}=0$.

In physical applications, such mass singularities are absorbed by the definition of the initial state "parton" densities and/or are canceled by the KLN theorem in the final state; we do not exponentiate them in the exactly mass less case.

We stress that the standard analytic properties of the 1PI 2 pt functions obtain here, as we use standard Feynman rules.
Wick rotation changes the Minkowski space Feynman loop integral $\int d^{4} k$ with $k=\left(k^{0}, k^{1}, k^{2}, k^{3}\right)$ for real $k^{j}$ and $k^{2}=k^{0^{2}}-$ $k^{1^{2}}-k^{2^{2}}-k^{3^{2}}$ into the integral $i \int d^{4} k_{E}$ with $k=\left(i k^{0}, k^{1}, k^{2}, k^{3}\right)$ and $k^{2}=-k^{0^{2}}-k^{1^{2}}-k^{2^{2}}-k^{3^{2}} \equiv-k_{E}^{2}$ with $k_{E}$ the Euclidean 4vector $k_{E}=\left(k^{0}, k^{1}, k^{2}, k^{3}\right)$ with metric $\delta_{\mu v}=\operatorname{diag}(1,1,1,1)$. Thus our results rigorously correspond to $\left|p^{2}\right|=-p^{2}$ in (48), (49) with $m^{2}$ replaced with $m^{2}-\mathrm{i} \varepsilon$, with $\varepsilon \downarrow 0$, following Feynman, for $p^{2}<0$; by Wick rotation this is the regime relevant to the UV behavior of the Feynman loop integral. Standard complex variables theory then uniquely specifies our exponent for any value of $p^{2}$.

\section{APPENDIX 2. EVALUATION OF GRAVITATIO- NALLY REGULATED LOOP INTEGRALS}

In this section we present the derivation of the representations which we have used in the text in evaluating the gravitationally regulated loop integrals in Figs. (2), (3).

Considering the integrals in Fig. (3) to show the methods, we need the result for

$$
\begin{aligned}
\mathcal{I}_{\bar{\mu} \bar{\nu} ; \mu \nu}= & i \frac{\int d^{4} k}{(2 \pi)^{4}} \frac{\left(k_{\bar{\mu}}^{\prime} k_{\bar{\nu}}+k_{\bar{\nu}}^{\prime} k_{\bar{\mu}}\right) e^{\frac{\kappa^{2}\left|k^{\prime 2}\right|}{8 \pi^{2}} \ln \left(\frac{m^{2}}{m^{2}+\left|k^{\prime 2}\right|}\right)}}{\left(k^{\prime 2}-m^{2}+i \epsilon\right)} \\
& \frac{\left(k_{\mu}^{\prime} k_{\nu}+k_{\nu}^{\prime} k_{\mu}\right) e^{\frac{\kappa^{2}\left|k^{2}\right|}{8 \pi^{2}} \ln \left(\frac{m^{2}}{m^{2}+\left|k^{2}\right|}\right)}}{\left(k^{2}-m^{2}+i \epsilon\right)} .
\end{aligned}
$$

In the limit that $\left|q^{2}\right|<M_{p l}^{2}$, standard symmetric integration methods give us, for the transverse parts,

$$
\mathcal{I}_{\bar{\mu} \bar{\nu} ; \mu \nu}=\frac{i \pi^{2}}{12}\left\{g_{\bar{\mu} \bar{\nu}} g_{\mu \nu}+\text { permutations }\right\} I_{0}
$$

where we have

$$
I_{0} \cong \frac{\int_{0}^{1} d \alpha \int_{0}^{\infty} d k k^{3}}{(2 \pi)^{4}} \frac{k^{4} e^{\lambda_{c}\left(k^{2} / m^{2}\right) \ln \left(m^{2} /\left(m^{2}+k^{2}\right)\right)}}{\left[k^{2}+m^{2}+\left|q^{2}\right| \alpha(1-\alpha)\right]^{2}}
$$

and where we used the symmetrization, valid under the respective integral sign,

$k_{\bar{\mu}} k_{\bar{\nu}} k_{\mu} k_{\nu} \rightarrow \frac{k^{4}}{24}\left\{g_{\bar{\mu} \bar{\nu}} g_{\mu \nu}+\right.$ permutations $\}$

and $\lambda_{c}=2 \mathrm{~m}^{2} /\left(\pi M_{p l}^{2}\right)$. The integral $I_{0}$, with the use of the mass counter-term, then leads us to evaluate the difference,

$\Delta I=I_{0}(q)-I_{0}(0) \cong \frac{\int_{0}^{1} d \alpha \int_{0}^{\infty} d x}{2(2 \pi)^{4}} \frac{x^{3}(x+1)^{-\lambda_{c} x}}{(x+1)^{2}(x+1+\bar{d})^{2}}\left(-2 \bar{d}(x+1)-\bar{d}^{2}\right)$

where we define here $\bar{d}=\left|q^{2}\right| \alpha(1-\alpha) / m^{2}$. It is seen that the dominant part of the integrals comes from the regime where $x \sim 1 /\left(\rho \lambda_{c}\right)$ with $\rho=-\ln \lambda_{c}$, so that we may finally write

$$
\begin{aligned}
\Delta I & =I_{0}(q)-I_{0}(0) \\
& \cong \frac{\int_{0}^{1} d \alpha \int_{0}^{\infty} d x}{2(2 \pi)^{4}} \frac{x^{3}(x+1)^{-\lambda x}}{(x+1)^{2}(x+1+\bar{d})^{2}}\left(-2 \bar{d}(x+1)-\bar{d}^{2}\right) \\
& \cong-\frac{|q|^{2} I_{1}}{6(2 \pi)^{4}}-\frac{|q|^{4} I_{2}}{60(2 \pi)^{4}}
\end{aligned}
$$


where we have defined

$$
\begin{aligned}
& I_{1}\left(\lambda_{c}\right)=\int_{0}^{\infty} d x x^{3}(1+x)^{-3-\lambda_{c} x}, \\
& I_{2}\left(\lambda_{c}\right)=\int_{0}^{\infty} d x x^{3}(1+x)^{-4-\lambda_{c} x} .
\end{aligned}
$$

The result (55) has been used in the text.

For the limit in practice, where we have $\lambda_{c} \rightarrow 0$, we can get accurate estimates for the integrals $I_{1}, I_{2}$ as follows. Consider first $\mathrm{I}_{2}$. Write $x^{3}=(x+1-1)^{3}=(x+1)^{3}-3(x+1)^{2}$ $+3(x+1)-1$ to get

$$
\begin{aligned}
I_{2}\left(\lambda_{c}\right) & =\int_{0}^{\infty} d x\left((1+x)^{-1}-3(x+1)^{-2}+3(x+1)^{-3}-(x+1)^{-4}\right)(1+x)^{-\lambda_{c} x} \\
& \cong \int_{0}^{\infty} d x(x+1)^{-1-\lambda_{c} x}-\frac{11}{6} .
\end{aligned}
$$

Use then the change of variable $r=\lambda_{c} x$ to get, for $\rho$ $=\ln \left(1 / \lambda_{c}\right)$,

$$
\begin{aligned}
& \int_{0}^{\infty} d x(x+1)^{-1-\lambda_{c} x}=\int_{0}^{\infty} d r \frac{e^{-r \ln \left(r+\lambda_{c}\right)-\rho r}}{r+\lambda_{c}} \\
& =-\ln \lambda_{c}+\int_{0}^{\infty} d r \ln \left(r+\lambda_{c}\right)\left(\ln \left(r+\lambda_{c}\right)+r /\left(r+\lambda_{c}\right)+\rho\right) e^{-r \ln \left(r+\lambda_{c}\right)-\rho r} \\
& \cong \rho+\left.\int_{0}^{\infty} d r \sum_{j=0}^{\infty} \frac{1}{j !}\left((\rho+1)(\partial / \partial \alpha)^{j+1}+(\partial / \partial \alpha)^{j+2}\right)(\partial / \partial \rho)^{j} r^{\alpha} e^{-\rho r}\right|_{\alpha=0} \\
& =\rho+\left.\sum_{j=0}^{\infty} \frac{1}{j !}\left((\rho+1)(\partial / \partial \alpha)^{j+1}+(\partial / \partial \alpha)^{j+2}\right)(\partial / \partial \rho)^{j} \Gamma(\alpha+1) \rho^{-\alpha-1}\right|_{\alpha=0} \\
& \cong \rho+\frac{-(\rho+1) \ln \rho+\ln ^{2} \rho}{\rho-\ln \rho} \\
& =\rho-\ln \rho-\frac{\ln \rho}{\rho-\ln \rho} .
\end{aligned}
$$

This gives us the approximation

$I_{2}\left(\lambda_{c}\right)=\rho-\ln \rho-\frac{\ln \rho}{\rho-\ln \rho}-\frac{11}{6}$

when $\lambda_{c} \rightarrow 0$, as we noted in the text.

The integral $I_{1}$ is a field renormalization constant so, in the usual renormalization program, we do not need it for most of the applications. Here, we will discuss it as well for completeness. We get

$$
\begin{aligned}
I_{1}\left(\lambda_{c}\right) & =\int_{0}^{\infty} d x(1+x)^{-\lambda_{c} x}-3\left(I_{2}\left(\lambda_{c}\right)+\frac{11}{6}\right)+\frac{5}{2} \\
& =\int_{0}^{\infty} d x(1+x)^{-\lambda_{c} x}-3 I_{2}\left(\lambda_{c}\right)-3,
\end{aligned}
$$

where, as above, we use

$$
\begin{aligned}
\int_{0}^{\infty} d x(1+x)^{-\lambda_{c} x} & =\frac{\int_{0}^{\infty} d r}{\lambda_{c}} e^{-r \ln \left(r+\lambda_{c}\right)-r \rho} \\
& \left.\cong \frac{\int_{0}^{\infty} d r}{\lambda_{c}} \sum_{j=0}^{\infty} \frac{1}{j !}(\partial / \partial \rho)^{j}(\partial / \partial \alpha)^{j} r^{\alpha} e^{-\rho r}\right|_{\alpha=0} \\
& =\left.\frac{1}{\lambda_{c}} \sum_{j=0}^{\infty} \frac{1}{j !}(\partial / \partial \rho)^{j}(\partial / \partial \alpha)^{j} \Gamma(1+\alpha) \rho^{-\alpha-1}\right|_{\alpha=0} \\
& \cong \frac{1}{\lambda_{c}} \frac{1}{\rho-\ln \rho} .
\end{aligned}
$$

Thus, we get

$$
I_{1}\left(\lambda_{c}\right) \cong \frac{1}{\lambda_{c}} \frac{1}{\rho-\ln \rho}-3 I_{2}\left(\lambda_{c}\right)-3 .
$$

Finally, let us show why we can neglect the terms $\bar{d}$ that were in the denominators of $I_{j}, j=1,2$. It is enough to look into the differences

$\Delta I_{j}=\frac{\int_{0}^{\infty} d x x^{3}}{(x+1)^{j}}\left(\frac{1}{(x+1)^{2}}-\frac{1}{(x+1+\bar{d})^{2}}\right)(x+1)^{-\lambda_{c} x}, j=1,2$

where we note that the integral $I_{1}$ is absorbed by the standard field renormalization where here for convenience we do this at $\left|q^{2}\right|=0$ when we neglect $\bar{d}$ in the denominator of $I_{1}$ or at the zero of the respective graviton propagator away from the origin otherwise. From this perspective, the main integral to examine to illustrate the level of our approximation becomes

$$
\begin{aligned}
& \Delta I_{2}=\frac{\int_{0}^{\infty} d x}{(x+1)^{2}}\left\{\frac{(x+1)^{-\lambda_{c} x}}{(x+1)^{2}}-\frac{(x+1)^{-\lambda_{c} x}}{(x+1+\bar{d})^{2}}\right\} \\
& =\frac{\int_{0}^{\infty} d r e^{-r \ln \left(r+\lambda_{c}\right)-r \rho}}{\left(r+\lambda_{c}\right)^{2}}\left\{\frac{1}{\left(r+\lambda_{c}\right)^{2}}-\frac{1}{\left(r+\lambda_{c}+\sigma\right)^{2}}\right\} \\
& \cong \int_{0}^{\infty} d r \int_{0}^{\infty} d \alpha_{1} \alpha_{1} \int_{0}^{\infty} d \alpha_{2} \alpha_{2} e^{-r \ln r-r \rho-\alpha_{1}\left(r+\lambda_{c}\right)-\alpha_{2}\left(r+\lambda_{c}\right)}\left(1-e^{-\alpha_{2} \sigma}\right),
\end{aligned}
$$

where we have defined $\sigma=\lambda_{c} \bar{d}$. The approximation, valid for small values of $\sigma$,

$$
\begin{aligned}
\left(1-e^{-\alpha_{2} \sigma}\right) & =2 e^{-\alpha_{2} \sigma / 2} \sinh \left(\alpha_{2} \sigma / 2\right) \\
& \cong \alpha_{2} \sigma e^{-\alpha_{2} \sigma / 2}
\end{aligned}
$$

then allows us to get

$$
\begin{aligned}
\Delta I_{2} & \cong 4 \sigma \frac{\partial^{2}}{\partial \sigma^{2}} \int_{0}^{\infty} d r e^{-r \rho}\left(1-\frac{\lambda_{c}+\sigma / 2}{r+\lambda_{c}+\sigma / 2}\right) \\
& \cong 2+\rho \sigma+2 \rho \sigma\left(1+\frac{1}{4} \rho \sigma\right) e^{\rho \sigma / 2}\left(C+\ln (\rho \sigma / 2)+\sum_{n=1}^{\infty} \frac{(-1)^{n}(\rho \sigma / 2)^{n}}{n n !}\right)
\end{aligned}
$$

which shows that this difference is indeed non-leading log. The analogous analysis holds for $\Delta I_{1}$ as well.

\section{APPENDIX 3: PROOF OF UV FINITENESS TO ALL ORDERS IN $\boldsymbol{\kappa}$}

For completeness, in this Appendix we review the proof given in Ref. [89] that the exponentially damped propagators we found in the text render QGR superficially UV finite to all orders in $\kappa$ as defined in the Introduction.

Let us examine the entire theory from (2) to all orders in $\kappa$ : we write it as

$\mathcal{L}(x)=\mathcal{L}_{0}(x)+\sum_{n=1}^{\infty} \kappa^{n} \mathcal{L}_{I}^{(n)}(x)$

in an obvious notation in which the first term is the free Lagrangian, including the free part of the gauge-fixing and ghost Lagrangians and the interactions, including the ghost interactions, are the terms of $\mathcal{O}\left(\kappa^{n}\right), n \geq 1$.

Each $\mathcal{L}_{I}^{(n)}$ is itself a finite sum of terms:

$\mathcal{L}_{I}^{(n)}(x)=\sum_{\ell=1}^{m_{n}} \mathcal{L}_{I, \ell}^{(n)}(x)$

$\mathcal{L}_{I}^{(n)}$ has dimension $d_{n, \ell} . d_{n}^{M} \max _{\ell}\left\{d_{n, \ell}\right\}$. As we have at least three fields at each vertex, the maximum power of 
momentum at any vertex in $\mathcal{L}_{I}^{(n)}$ is $=\bar{d}_{n}^{M} \min \left\{d_{n}^{M}-3,2\right\}$ and is finite (here, we use the fact that the Riemann tensor is only second order in derivatives). We will use this fact shortly.

First we stress that, in any gauge, if $P_{\alpha_{1} \cdots ; \alpha_{1}^{\prime} \cdots}$ is the respective propagator polarization sum for a spinning particle, then the spin independence of the soft graviton resummation exponential factoring (22) yields the respective resumed improved Born propagator as

$\left.i \mathcal{D}_{F \alpha_{1} \cdots ; \alpha_{1}^{\prime} \cdots}^{(0)}(k)\right|_{\text {resummed }}=\frac{i P_{\alpha_{1} \cdots ; \alpha_{1}^{\prime} \cdots} e^{B_{g}^{\prime \prime}(k)}}{\left(k^{2}-m^{2}+i \epsilon\right)}$,

so that it is also exponentially damped at high energy in the deep Euclidean regime (DER). Our improved Born propagators are then used throughout the respective resummed loop expansion according to the standard resummation algebra well-tested in the electroweak theory $[98,148]^{15}$. We will use this shortly as well.

Now consider any one particle irreducible vertex $\Gamma_{N}$ with $[N] \equiv n_{1}+n_{2}$ amputated external legs, where we use the notation $N=\left(n_{1}, n_{2}\right)$, when $n_{1}\left(n_{2}\right)$ is the respective number of graviton (scalar) external lines. We always assume we have Wick rotated. At its zero-loop order, there are only tree contributions which are manifestly UV finite. Consider the first loop $\left(\mathcal{O}\left(\kappa^{2}\right)\right)$ corrections to $\Gamma_{N}$. There must be at least one improved exponentially damped propagator in the respective loop contribution and at most two vertices so that the maximum power of momentum in the numerator of the loop due to the vertices is $\max \left\{2 \bar{d}_{1}{ }^{M}, 2 \bar{d}_{2}{ }^{M}\right\}$ and is finite. The exponentially damped propagator then renders the loop integrals finite and as there are only a finite number of them, the entire one-loop $\left(\mathcal{O}\left(\kappa^{2}\right)\right)$ contribution is finite.

As a corollary, if $\Gamma_{N}$ vanishes in tree approximation, we can conclude that its first non-trivial contributions at oneloop are all finite, as in each such loop the exponentially damped propagator which must be present is sufficient to damp the respective finite order polynomial in loop momentum that occurs from its vertices by our arguments above into a convergent integral.

As an induction hypothesis suppose all contributions to all $\left\{\Gamma_{N}\right\}$ for m-loop corrections $\left(\mathcal{O}\left(\kappa^{2 m}\right)\right), m<n$, are finite. At the n-loop $\left(\mathcal{O}\left(\kappa^{2 n}\right)\right)$ level, when the exponentially damped improved Born propagators are taken into account, we argue that respective n-loop integrals are finite as follows. First, by momentum conservation, if $\left\{\ell_{1}, \ldots, \ell_{n}\right\}$ are the respective Euclidean loop momenta, we may without loss of content assume that $\ell_{n}$ is precisely the momentum of one of the exponentially damped improved Born propagators. The $n-$ 1 loop integrations over the remaining loop variables $\left\{\ell_{1}, \ldots\right.$, $\left.\ell_{n-1}\right\}$ for fixed $\ell_{n}$ then produces the contribution of a subgraph which if it is 1PI is a part of $\Gamma_{N+2}$ and which if it is not 1PI is a product of the contributions to the respective $\left\{\Gamma_{J}\right\}$ and the respective improved resummed Born propagator functions. This is then finite by the induction hypothesis. Here, $N+2=$

${ }^{15}$ This applies both to the graviton and to its ghosts in Feynman's gauge. $\left(n_{1}+2, n_{2}\right)\left(\left(n_{1}, n_{2}+2\right)\right)$ according as the propagator with momentum $\ell_{n}$ which we fix as multiplying the remaining subgraph is a graviton (scalar) propagator, respectively. The application of standard arguments [149] from Lebesgue integration theory (specifically, for any two measurable functions $f, g, f \leq g$ almost everywhere implies that $\int f \leq g$ ) in conjunction with Weinberg's theorem $[150,151]$ guarantees that this finite result behaves at most as a finite power of $\left|\ell_{\mathrm{n}}\right|$ modulo Weinberg's logarithms for $\left|\ell_{n}\right| \rightarrow \infty$. It follows that the remaining integration over $\ell_{n}$ is damped into convergence by the already identified exponentially damped propagator with momentum $\ell_{n}$. Thus, each n-loop contribution to $\Gamma_{N}$ is finite, from which it follows that $\Gamma_{N}$ is finite at $n$-loop level. Pictorially, we illustrate the type of situations we have in Fig. (4).

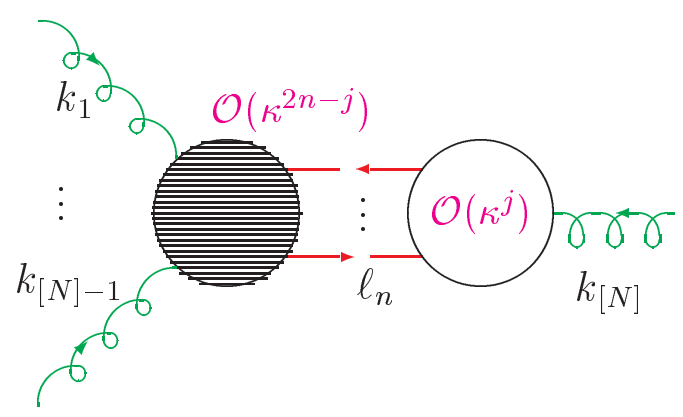

Fig. (4). The typical contribution we encounter in $\Gamma_{N}$ at the n-loop level; $\ell_{n}$ is the $n$-th loop momentum and is precisely the momentum of the indicated resummed improved Born propagator.

We conclude by induction that all $\left\{\Gamma_{N}\right\}$ in our theory are finite to all orders in the loop expansion. Of course, the sum of the respective seriesin $\kappa$ may very well not actually converge but this issue is beyond the scope of our work.

This completes our Appendix.

\section{LIST OF ABBREVIATIONS}

$\begin{array}{ll}\text { CERN } & \text { European Laboratory for Elementary Particle } \\ & \text { Physics } \\ \text { CL } & \text { Collinear } \\ \text { IR } & \text { Infrared } \\ \text { LEP } & \text { Large Electron Positron } \\ \text { LHC }= & \text { Large Hadron Collider } \\ \text { QCD }= & \text { Quantum chromodynamics } \\ \text { QED }= & \text { Quantum electrodynamics } \\ \text { QGR }= & \text { Quantum general relativity } \\ \text { SM }= & \text { Standard Model } \\ \text { UV }= & \text { Ultraviolet } \\ \text { YFS }= & \text { Yennie-Frautschi-Suura }\end{array}$

\section{REFERENCES}

[1] Misner C, Thorne KS, Wheeler JA. Gravitation. Freeman: San Francisco 1973. 
[2] Weinberg S. Gravitation and cosmology: principles and applications of the general theory of relativity. John Wiley: New York, 1972.

[3] Glashow SL. Partial symmetries of weak interactions. Nucl Phys 1961; 22: 579-88

[4] Weinberg S. A model of leptons. Phys Rev Lett 1967; 19: 1264-6.

[5] Salam, A. Elementary particle theory. In: Svartholm N, Ed. Almqvist and Wiksells, Stockholm, 1968; p. 367.

[6] 't Hooft G, Veltman M. Regularization and renormalization of gauge fields. Nucl Phys B 1972; 44: 189-213.

[7] 't Hooft G, Veltman M. Combinatorics of gauge fields. Nucl Phys B 1972; 50: 318-53.

[8] 't Hooft G. Renormalizable Lagrangians for massive Yang-Mills fields. Nucl Phys B 1971; 35: 167-88.

[9] Veltman M. Perturbation theory for massive Ynag-Mills fields. Nucl Phys B 1968; 7: 637-50.

[10] Gross DJ, Wilczek F. Ultraviolet behavior of nonabelian gauge theories. Phys Rev Lett 1973; 30: 1343-46.

[11] Politzer HD. Reliable perturbative results for strong interactions? Phys Rev Lett 1973; 30: 1346-49.

[12] Wilczek F. Status of QCD. In: Drell P, Rubin DL, Eds. Proceedings of $16^{\text {th }}$ International Symposium on Lepton and Photon Interactions, AIP, New York 1993: pp. 593-633.

[13] Yang CN, Mills RL. Conservation of isotopic spin and isotopic gauge in variance. Phys Rev 1954; 96: 191-5.

[14] Abbaneo D, Ainsly Ch, Alcaraz J, et al. A Combination of preliminary electroweak measurements and constraints on the Standard Model. 1 Apr 2003. Available from: http://www.arxiv. org/ abs/hep-ex/0212036

[15] Gruenewald MW. Electroweak Physics. In: Bentvelsen S, de Jong P, Koch J, Laenen E, Eds. Proceedings of the $31^{\text {st }}$ International Conference on High Energy Physics, North-Holland, Amsterdam, Nucl Phys B (Proc Suppl) 2003; vol. 117: pp. 280-97.

[16] Royal Swedish Academy. Press Release: the 1999 Nobel Prize in Physics. 12 October, 1999. Available from: http://nobelprize.org/ nobel prizes/physics/laureates/1999/press.html

[17] Green M, Schwarz J, Witten E. Superstring theory. Cambridge Univ Press: Cambridge 1987; vol. 1, vol. 2.

[18] Polchinski J. String theory. Cambridge Univ Press: Cambridge 1998; vol. 1, vol. 2.

[19] Thiemann T. Loop quantum gravity. In: Zambrini J-C, Ed. Proceedings of $14^{\text {th }}$ International Congress on mathematical Physics, World Scientific Publ, Hackensack 2005; pp. 569-83.

[20] Smolin L. How far are we from the quantum theory of gravity? Available from: http://www.arxiv.org/abs/hep-th/0303185.

[21] Ashtekar A, Lewandowski J. Background independent quantum gravity: a status report. Class Quantum Grav 2004; 21: R53-153.

[22] Feynman RP. Quantumtheory of gravitation. Acta Phys Pol 1963; 24: 697-722.

[23] Feynman RP. Feynman lectures on gravitation. Moringo FB, Wagner WG, Eds. Caltech, Pasadena 1971.

[24] Gross DJ. The triumph and limitations of quantum field theory. In: Cao TY, Ed. Proc. Conceptual Foundations of Quantum Field Theory, Cambridge Univ Press, Cambridge 1999; pp. 56-67.

[25] Wheeler JA. Relativity, groups and topology, Gordon and Breach, In: DeWitt BS, DeWitt CM, Eds. New York 1963: p. 315.

[26] Sterman G. Summation of large corrections to short distance hadronic cross-sections. Nucl Phys B 1987; 281: 310-64.

[27] Catani S, Trentadue L. Resummation of the QCD perturbative series for hard processes. Nucl Phys B 1989; 327: 323-52.

[28] Catani S, Trentadue L. Comment on QCD exponentiation at large x. Nucl Phys B 1991; 353: 183-6.

[29] Collins JC, Soper DE, Sterman G. Transverse momentum distribution in Drell-Yan pair and $\mathrm{W}$ and $\mathrm{Z}$ boson production. Nucl Phys B 1985; 250: 199-224.

[30] Jackson JD, Scharre DL. Initial state radiative and resolution corrections and resonance parameters in $\mathrm{e}+\mathrm{e}$-annihilation. Nucl Instrum Meth 1975; 128: 13-23.

[31] Jackson JD. Radiative corrections and resonance parameters in e+e-annihilation. In: Bjorken JD, Brodsky S, Budny R, et al, Eds. Notes from the SLAC Theory Workshop on the psi Concerning: Interference Effects, Angular Distributions, How to Extract Widths, Radiative Corrections. SLAC-PUB-1515 1974; pp. 21-37. Available from: http://www.slac.stanford.edu/pubs/slacpubs/1000/ slac-pub-1515.html
[32] Tsai Y-S. $\psi$ : Extraction of decay widths and coupling constants. How to deal with radiative corrections and machine energy width. In: Bjorken JD , Brodsky S, Budny R, et al, Eds. Notes from the SLAC Theory Workshop on the psi Concerning: Interference Effects, Angular Distributions, How to Extract Widths, Radiative Corrections. SLAC-PUB-1515 1974; pp. 38-48. Available from http://www.slac.stanford.edu/pubs/slacpubs/1000/ slac-pub-1515.html

[33] Yennie DR, Frautschi SC, Suura H. The infrared divergence phenomena and high energy processes. Ann Phys 1961; 13: 379452.

[34] Mahanthappa KT. Multiple production of photons in quantum electrodynamics. Phys Rev 1962; 126: 329-40.

[35] Jadach S, Ward BFL. Exponentiation of soft photons in the Monte Carlo: the case of Bonneau and Martin. Phys Rev D 1988; 38: 2897-903.

[36] Jadach S, Ward BFL. Erratum: exponentiation of soft photons in Monte Carlo event generators: The case of the Bonneau-Martin cross section. Phys Rev D 1989; 39: 1472.

[37] Jadach S, Ward BFL. Multiphoton Monte Carlo event generator for Bhabha scattering at small angles. Phys Rev D 1989; 40: 3582-89.

[38] Jadach S, Ward BFL. YFS2: the second order Monte Carlo for fermion pair production atLep/ Slc withtheinitial state radiationoftwohard and multiple soft photons. Comput Phys Commun 1990; 56: 351-84.

[39] Jadach S, Ward BFL. Final state multiple photon effects in fermion pair production at SLC/ LEP. Phys Lett B 1992; 274: 470-2.

[40] Jadach S, Richter-Was E, Ward BFL, Was Z. Monte Carlo program BHLUMI-2.01 for Bhabha scattering at low angles with YennieFrautschi-Suura exponentiation. Comput Phys Commun 1992; 70:305-344.

[41] Jadach S, Placzek W, Richter-Was E, Ward BFL, Was Z. Upgrade of the Monte Carlo program BHLUMI for Bhabha scattering at low angles to version 4.04. Comput Phys Commun 1997; 102: 229-51.

[42] Jadach S, Placzek W, Ward BFL. BHWIDE 1.00: $\mathcal{O}$ (alpha) YFS exponentiated Monte Carlo for Bhabha scattering at wide angles for LEP-1 / SLC and LEP-2. Phys Lett B 1997; 390: 298-308.

[43] Jadach S, Skrzypek M, Ward BFL. Soft pairs corrections to low angle Bhabha scattering: YFS Monte Carlo approach. Phys Rev D 1997; 55: 1206-15.

[44] Jadach S, Placzek W, Ward BFL. Gaugeinvariant YFS exponentiation of (un)stable $\mathrm{Z}$ pair production at and beyond LEP-2 energies. Phys Rev D 1997; 56: 6939-41.

[45] Jadach S, Placzek W, Skrzypek M, Ward BFL, Was Z. Exact $\mathcal{O}$ (alpha) gauge invariantYFS exponentiated Monte Carlofor (un)stable $\mathrm{W}+\mathrm{W}$-production atand beyond LEP-2 energies. Phys Lett B 1998; 417: 326-36.

[46] Jadach S, Ward BFL, Was Z. Coherent exclusive exponentiation for precision Monte Carlo calculations. Phys Rev D 2001; 63: 113009 .

[47] Jadach S, Ward BFL, Was Z. The Precision Monte Carlo event generator $\mathrm{K} \mathrm{K}$ for two fermion final states in e+ e-collisions. Comput Phys Commun 2000; 130: 260-325.

[48] Jadach S, Placzek W, Skrzypek M, Ward BFL, Was Z. The Monte Carlo event generatorYFSWW3 version1.16forWpairproduction anddecay atLEP-2/ LC energies. Comput Phys Commun 2001; 140: $432-74$.

[49] Jadach S, Placzek W, Skrzypek M, Ward BFL, Was Z. The Monte Carlo program KoralW version 1.51 and the concurrent Monte Carlo KoralW and YFSWW3 with allbackgroundgraphsandfirst ordercorrectionstoWpairproduction.Comput Phys Commun 2001; 140: 475-512.

[50] Jadach S, Skrzypek M, Ward BFL. Analytical results for low angle Bhabha scattering with pair production. Phys Rev D 1993; 47: 3733-41.

[51] Jadach S, Skrzypek M, Ward BFL. Is there a better way of exponentiating QED corrections? Phys Lett B 1991; 257: 173-8.

[52] Jadach S, Skrzypek M, Ward BFL. Exponentiation, higher orders and leading logs. In: Van JTT, Ed. $\mathrm{Z}^{\mathrm{O}}$ Physics: Proceedings of $\mathrm{XXV}^{\text {th }}$ Rencontre de Moriond, Les Arcs, France, Editions Frontieres, Gif-Sur-Yvette 1990.

[53] Jadach S, Richter-Was E, Ward BFL, Was Z. High precision improved analytic exponentiation results for multiple photon effects in low angle Bhabha scattering at SLC and LEP. Phys Rev D 1991; 44: 2669-77. 
[54] Jadach S, Ward BFL. Exclusive exponentiation in the Monte Carlo Yennie-Frautschi-Suura approach. In: Dombey N, Boudjema F, Eds. Proceedings Brighton Workshop, Plenum, London 1990; pp. 325-40.

[55] Ward BFL, Jadach S. YFS MC approach to QCD soft gluon exponentiation. Acta Phys Polon 2002; B33: 1543-58.

[56] Ward BFL, Jadach S. QCD soft gluon exponentiation: YFS MC approach. In: Bentvelsen S, de Jong P, Koch J, Laenen E, Eds. Proceedings of the $31^{\text {st }}$ International Conference on High Energy Physics, North-Holland, Amsterdam 2003; pp. 275-78.

[57] Ward BFL, Jadach S. Dokshitzer-Gribov-Lipatov-Altarelli-Parisi evolution and the renormalization group improved YennieFrautschi-Suura theory inQCD. Mod Phys Lett A 1999; 14: 491500 .

[58] DeLaney DB, Jadach S, Shio C, Siopsis G, Ward BFL. Multiple gluon effects in $\mathrm{q}+$ anti- $\mathrm{q} \rightarrow \mathrm{t}+$ anti- $\mathrm{t}+\mathrm{X}$ at FNAL energies: Semianalytical results. Mod Phys Lett A 1997; 12: 2425-34.

[59] DeLaney DB, Jadach S, Shio C, Siopsis G, Ward BFL. Multiple gluon effects in fermion anti-fermion scattering atSSC/CERNLHC energies. Phys Rev D 1995; 52: 108-15.

[60] DeLaney DB, Jadach S, Shio C, Siopsis G, Ward BFL. Erratum: multiple gluon effects in fermion-(anti) fermion scattering at and beyond CERN LHC energies. Phys Rev D 2002; 66: 019903.

[61] DeLaney DB, Jadach S, Shio C, Siopsis G, Ward BFL. Renormalization group improved exponentiation of softgluonsin QCD. Phys Lett B 1995; 342: 239-44.

[62] Glosser C, Jadach S, Ward BFL, Yost SA. QED x QCD threshold corrections atthe LHC. Mod Phys Lett A 2004; 19: 2113-20.

[63] Ward BFL, Glosser C, Jadach S, Yost SA. Threshold corrections in precision LHC physics:QED x QCD. Int J Mod Phys A 2005; 20: 3735-38.

[64] Ward BFL, Glosser C, Jadach S, Yost SA. Threshold corrections in QED x QCD at the LHC. In: Chen H, Du D, Li W, Lu C, Eds. Proceedings of Beijing 2004, ICHEP 2004, World Sci Publ, Singapore 2005; vol. 1: pp. 588-91.

[65] Ward BFL, Yost S. QED x QCD exponentiation and shower/ME matching at the LHC. In: De Roeck A, Jung H, Eds. HERA and the LHC: A Workshop on the implications of HERA for LHC physics: Proceedings Parts A, B, CERN, Geneva 2005; pp. 304-8.

[66] Bethke S. as at Zinnowitz 2004. Nucl Phys B (Proc Suppl) 2004; 135: 345-52.

[67] See for example Gwenlan C. Deep inelastic scattering at HERA. In: Son D, Oh SK, Eds. Proceedings of LP07, in press.

[68] Weinberg S. Ultraviolet divergences in quantum theories of gravitation. In: Hawking SW, Israel W, Eds. General relativity. Cambridge Univ Press, Cambridge 1979; pp.790-831.

[69] Freedman DZ, van Nieuwenhuizen P, Ferraro S. Progress toward a theory of super-gravity. Phys Rev D 1976; 13: 3214-18.

[70] Lauscher O, Reuter M. Flow equation of quantum Einstein gravity in a higher-derivative truncation. Phys Rev D 2002; 66: 025026.

[71] Bonnanno A, Reuter M. Renormalization group improved black hole spacetimes. Phys Rev D 2000; 62: 043008.

[72] Litim D. Fixed points of quantum gravity. Phys Rev Lett 2004; 92 : 201301.

[73] Litim D. Optimized renormalization group flows. Phys Rev D 2001; 64: 105007 .

[74] Percacci R, Perini D. Asymptotic safety of gravity coupled to matter. Phys Rev D 2003; 68: 044018.

[75] Donoghue J. Leading quantum correction to the Newtonian potential. Phys Rev Lett 1994; 72: 2996-9.

[76] Donoghue J. General relativity as an effective field theory: the leading quantum corrections. Phys Rev D 1994; 50: 3874-88.

[77] Donoghue J. Introduction to the effective field theory description of gravity. Available from: http://www.arxiv.org/abs/gr-qc/9512024

[78] Donoghue J, Holstein BR, Garbrecht B, Konstandin T. Quantum corrections to the Reissner-Nordstrom and Kerr-Newman metrics. Phys Lett B 2002; 529: 132-42.

[79] Cavaglia M, Fabbri A. Quantum gravitational corrections to black hole geometries. Phys Rev D 2002; 65: 044012.

[80] Cavaglia M, Ungarelli C. Quantum gravity corrections to the Schwarzschild mass. Phys Rev D 2000; 61: 064019.

[81] Shapiro I, Sola J. On the scaling behavior of the cosmological constant and the possible existence of new forces and new light degrees of freedom. Phys Lett B 2000; 475: 236-46.
[82] Shapiro I, Sola J. Scaling behavior of the cosmological constant: interface between quantum field theory and cosmology. J High Energy Phys 2002; 0202: 006.

[83] Espana-Bonet C, Ruiz-Lapuente P, Shapiro IL, Sola J. Testing the running of the cosmological constant with type Ia supernovae at high z. J Cos Astropart Phys 2004; 0402: 006.

[84] Shapiro IL, Sola J, Espana-Bonet C, Ruiz-Lapuente P. Variable cosmological constant as a Planck scale effect. Phys Lett B 2003; 574: $149-55$

[85] Shapiro I, Sola J, Stefancic, H. Running G and Lambda at low energies from physics at $\mathrm{M}(\mathrm{X})$ : possible cosmological and astrophysical implications. J Cos Astropart Phys 2005; 0501: 012.

[86] Hawking SW. Blackhole explosions. Nature (London) 1974; 248 : 30-1.

[87] Hawking SW. Particle creation by black holes. Commun Math Phys 1975; 43: 199-220.

[88] Hawking SW. Erratum: particle creation by black holes. Commun Math Phys 1976; 46: 206.

[89] Ward BFL. Quantum corrections to Newton's law. Mod Phys Lett A $2002 ; 17: 2371-82$.

[90] Ward BFL. Are massive elementary particles black holes? Mod Phys Lett A 2004; 19: 143-50.

[91] Ward BFL. Massive elementary particles and black holes. J Cos Astropart Phys 2004; 0402: 011 .

[92] Ward BFL. Planck scale remnants in resummed quantum gravity. Acta Phys Polon B 2006; 37: 1967-74.

[93] Ward BFL. Final state of Hawking radiation in quantum general relativity. Acta Phys Polon B 2006; 37: 347-56.

[94] Ward BFL. Black holes and massive elementary particles in resummed quantum gravity. In: Kreitler PV, Ed. Focus on black hole research, Nova, New York 2006.

[95] Ward BFL. Quantum corrections to Newton's law in resumed quantum gravity. Int J Mod Phys A 2005; 20: 3502-6.

[96] Ward BFL. Massive elementary particles and black hole physics in resummed quantum gravity. Int J Mod Phys A 2005; 20: 3128-31.

[97] Ward BFL. Massive elementary particles and black holes in resummed quantum gravity. In: Chen $\mathrm{H}, \mathrm{Du} \mathrm{D}, \mathrm{Li} \mathrm{W}, \mathrm{Lu} \mathrm{C}, \mathrm{Eds}$ Proc. Beijing 2004, ICHEP 2004, World Sci Publ, Singapore 2005; vol. 1: pp. 419-22.

[98] Bardin D, Passarino G. The standard model in the making: precision study of the electroweak interactions. Oxford Univ Press: London 1999.

[99] Gianotti F. Probing the hierarchy problem with the LHC. In: Brenner R, de los Heros P, Rathsman J, Eds. Proceedings of LP2005, World Sci Publ Co, Singapore 2006; pp. 54-65.

[100] Engelen J. Physics at the LHC with ATLAS and CMS. In: Blumlein J, Moch S, Riemann T, Eds. Proceedings of $7^{\text {th }}$ DESY Workshop on Elementary Particle Theory: Loops and Legs in Quantum Field Theory, Elsevier, Amsterdam Nucl Phys B (Proc Suppl) 2004; 135: pp. 3-8.

[101] Perlmutter S, Aldering G, Goldhaber G, et al. Measurements of Omegaand Lambda from 42 high redshift supernovae. Astrophys J 1999; 517: 565-86.

[102] Faddeev LD, Popov VN. Perturbation theory for gauge invariant fields. Preprint ITF-67-036, NAL-THY-57 (translated from Russian by Gordon D and Lee BW). Available from: http://lss.fnal.gov/archive/test-preprint/fermilab-pub-72-057t.shtml

[103] Faddeev LD, Popov VN. Feynman diagrams for the Yang-Mills field. Phys Lett B 1967; 25: 29-30.

[104] Dokshitzer Y, Khoze VA, Troian SI, Mueller A. QCD coherence in high-energy reactions. Rev Mod Phys 1988; 60: 373-88.

[105] Weinberg S. The quantum theory of fields, I. Cambridge: Cambridge 1995.

[106] Weinberg S. The quantum theory of fields, III. Cambridge: Cambridge 2000.

[107] Ellis RK, Georgi H, Machacek M, Politzer HD, Ross GG. Factorization and the parton modelin QCD. Phys Lett B 1978; 78: 281-4.

[108] Ellis RK, Georgi H, Machacek M, Politzer HD, Ross GG. Perturbation theory and the parton modelin QCD. Nucl Phys B 1979; 152: 285-329.

[109] Amati D, Petronzio R, Veneziano G. Relating hard QCD processes through universality of mass singularities (II). Nucl Phys B 1978; 146: $29-49$. 
[110] Libby S, Sterman G. Jet and lepton-pair production in high-energy lepton-hadron and hadron-hadron scattering. Phys Rev D 1978; 18 : 3252-68.

[111] Mueller A. Cut vertices and their renormalization: a generalization of the Wilson expansion. Phys Rev D 1978; 18: 3705-27.

[112] Callan CG, Jr. Broken scale invariance in scalar field theory. Phys Rev D 1970; 2: 1541-7.

[113] Symanzik K. Small distance behavior in field theory and power counting. Commun Math Phys 1970; 18: 227-46.

[114] Symanzik K. Small-distance behaviour in field theory. In: Hoehler G, Ed. Springer Tracts in Modern Physics, Springer, Berlin 1971; vol. 57: pp. 222-36.

[115] Novello M, Neves RP. The mass of the graviton and the cosmological constant. Class Quantum Grav 2003; 20: L67-73.

[116] Novello M, Neves RP. Spin-2 field theory in curved space-time in the Fierz representation. Class Quantum Grav 2002; 19: 5335-51.

[117] Gazeau JP, Novello $M$. The nature of $\Lambda$ and the mass of the graviton: a critical view. Available from: http://www.arxiv.org/ $\mathrm{abs} / \mathrm{gr}-\mathrm{qc} / 0610054$

[118] Novello M. The mass of the graviton and the cosmological constant puzzle. In: Neto JAH, Bytsenko AA, Eds. Proceedings of $5^{\text {th }}$ International Conference on Mathematical Methods in Physics, PoS IC 2006; 009 .

[119] 't Hofft G, Veltman M. One-loop divergencies in the theory of gravitation. Ann Inst Henri Poincare 1974; 20: 69-94.

[120] vanDam H, Veltman M. Massive and mass-lessYang-Mills and gravitational fields. Nucl Phys B 1970; 22: 397-411.

[121] Zakharov VI. Linearized gravitation theory and the graviton mass. Pisma Zh Eksp Teor Fiz 1970; 12: 447-9.

[122] See for example Wark DL. Neutrino masses and mixings experiments past, present, and future. In: Bentvelsen S, de Jong P, Koch K, Laenen E, Eds. Proc. ICHEP02, North-Holland, Amsterdam, Nucl Phys B (Proc Suppl) 2003; 117: pp. 164-85.

[123] Gonzalez-Garcia MC. Theory of neutrino masses and mixings. In: Bentvelsen S, de Jong P, Koch K, Laenen E, Eds. Proceedings of ICHEP02, North-Holland, Amsterdam, 2003 Nucl Phys B (Proc Suppl) 2003; 117: pp. 186-203.

[124] Hagiwara K, Hikasa K, Nakamura K, et al. Review of particle properties. Phys Rev D 2002; 66: 010001.

[125] Leutwyler H, Gasser J. Quark masses. Phys Rep 1982; 87: 77-169.

[126] Eidelman S, Hayes KG, Olive KA, et al. Review of particle physics. Phys Lett B 2004; 592: 1-1109.

[127] Kalmykov M Yu, Kazakov DI. Calculation of the off-shell renormalization functions in $\mathrm{R}^{2}$ gravity. Phys Lett B 1997; 404: 253-8.

[128] Buchbinder IL, Odintsov SD, Shapiro IL. Effective Actionin Quantum Gravity. IOP Publ Co: Bristol and Philadelphia 1992.

[129] Bojowald M, Goswami R, Maartens R, Singh P. A black hole mass threshold from non-singular quantum gravitational collapse. Phys Rev Lett 2005; 95: 091302.

[130] Falkenberg S, Odintsov SD. Gauge dependence of the effective average action in Einstein gravity. Int J Mod Phys A 1998; 13: 60723

[131] Rizzo TG. TeV-scale black holes in warped highercurvaturegravity. Available from: http://www.arxiv.org/abs/hep$\mathrm{ph} / 0510420$

Received: November 21, 2008

Revised: February 06, 2009
[132] Rizzo TG. TeV-scale black hole lifetimes in extra-dimensional Lovelock gravity. Class Quantum Grav 2006; 23: 4263-80.

[133] Rizzo TG. Noncommutative inspired black holes in extra dimensions. J High Energy Phys 2006; 0609: 021 - in Refs. [131133] Rizzo also finds that certain higher dimensional $\mathrm{TeV}$-scale gravity theories lead to Planck-scale remnants via the Hawking process.

[134] Linsley J. Evidence for a primary cosmic-ray particle with energy $10^{2} 0$ eV. Phys Rev Lett 1963; 10: 146-8.

[135] Linsley J. Catalogue of the highest energy cosmic rays, Volcano Ranch (1980) No. 1, World Data Center C2 for Cosmic Rays, Institute of Physical and Chemical Research, Itabashi, Tokyo 1980; pp. 3-59.

[136] Efimov NN, Egorov TA, Glushkov AV, Pravdin MI, Sleptsov IE. The energy spectrum and anisotropy of primary cosmic rays at energy $\mathrm{E}_{0}>10^{17}-\mathrm{eV}$ observed in Yakutsk. In: Nagano M, Takahara F, Eds. Astrophysical Aspects of the Most Energetic Cosmic Rays, World Scientific Publ Co, Singapore 1991; pp. 20-33.

[137] Bird DJ, Corbato SC, Dai HY, et al. Evidence for correlated changes in the spectrum and composition of cosmic rays at extremely high energies. Phys Rev Lett 1993; 71: 3401-4.

[138] Bird DJ, Corbato SC, Dai HY, et al. The Cosmic ray energy spectrum observed by the Fly’s Eye. Astrophys J 1994; 424: 491502 .

[139] Takeda M, Hayashida N, Honda K, et al. Extension of the cosmicray Energy spectrum beyond the predicted Greisen-ZatsepinKuz'min cutoff. Phys Rev Lett 1998; 81: 1163-6.

[140] Takeda M, Hayashida N, Honda K, et al. Small-scale anisotropy of cosmic rays above $10^{1} 9 \mathrm{eV}$ observed with the Akeno Giant Air Shower Array. Astrophys J 1999; 522: 225-37.

[141] Brenner R, de los Heros CP, Rathsman J, Eds. For a review of the current UHECR data, Westerhoff $\mathrm{S}$. Ultra-high-energy cosmic rays. Proc. LP2005, World Sci Publ Co, Singapore 2006; pp. 376-87.

[142] Hawking SW. Black holes and the information paradox. In: Florides P, Nolan B, Ottewill A, Eds. Proceedings of GR17, World Sci Publ Co, Hackensack 2005; pp. 56-62.

[143] Ferrara S, Freedman DZ, van Nieuwenhuizen P. Progress toward a theory of super-gravity. Phys Rev D 1976; 13: 3214-8.

[144] Freedman DZ, van Nieuwenhuizen P. Properties of supergravity theory. Phys Rev D 1976; 14: 912-6.

[145] van Nieuwenhuizen P. Supergravity. Phys Rep 1981; 68: 189-398.

[146] Kreimer D. A remark on quantum gravity. Ann Phys 2008; 323: 49-60.

[147] Kreimer D. Anatomy of a gauge theory. Ann Phys 2006; 321: 2757-81.

[148] Altarelli G, Kleiss R, Verzegnassi C. Z Physics at LEP1, CERN89-08. CERN: Geneva 1989; vol. 1-3.

[149] Royden HL. Real analysis. MacMillan Co: New York 1968.

[150] Weinberg S. High-energy behavior in quantum field theory. Phys Rev 1960; 118: 838.

[151] Hepp K. Proof of the Bogolyubov-Parasiuk theorem on renormalization. Commun Math Phys 1966; 2: 301-26.

(C) B.F.L. Ward; Licensee Bentham Open.

This is an open access article licensed under the terms of the Creative Commons Attribution Non-Commercial License (http://creativecommons.org/licenses/by$\mathrm{nc} / 3.0 /$ ), which permits unrestricted, non-commercial use, distribution and reproduction in any medium, provided the work is properly cited. 